\title{
Linear and nonlinear microrheometry of small samples and interfaces using microfabricated probes
}

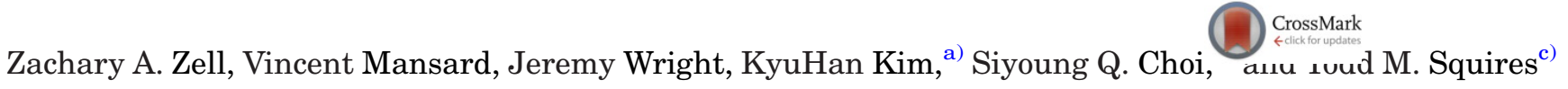 \\ Department of Chemical Engineering, University of California, Santa Barbara, California 93106-5080
}

(Received 24 August 2015; final revision received 1 December 2015; published 24 December 2015)

\begin{abstract}
We describe a microrheological strategy that enables sensitive surface shear rheology measurements of surfactant-laden interfaces, with the capacity to simultaneously visualize deforming interfaces. This technique utilizes a ferromagnetic microbutton probe pinned to a fluid-fluid interface, and actively torqued or forced with externally controlled electromagnets. Various modes of operation are possible: Smallamplitude oscillatory rotations, which provide frequency-dependent viscoelastic shear moduli; controlled torque (analogous to fixing shear stress); controlled rotation rate (analogous to fixing strain rate); and imposed force (analogous to active, translational microrheology). The circular shape of the probe ensures pure shear strains (when driven to rotate). We describe the experimental apparatus, its measurement limits and sources of error. We then highlight its versatility and capabilities with measurements on a variety of qualitatively distinct systems, including purely viscous monolayers, block-copolymer interfaces, aging and evolving interfaces, colloidal monolayers, and bulk rheometry of Newtonian and viscoelastic materials, with sample volumes as small as $2 \mu \mathrm{l}$. C 2016 The Society of Rheology.

[http://dx.doi.org/10.1122/1.4937931]
\end{abstract}

\section{INTRODUCTION}

Surface-active materials, including small amphiphilic molecules, copolymers, proteins, nanoparticles, and colloids, are used throughout engineering and science to modify both the static and dynamic properties of fluid-fluid interfaces. Surfactants are found in a broad spectrum of multiphase materials important to the food, personal care, biomedical, and petroleum industries [1,2], in addition to biological and soft matter physics [3].

While their static properties have long been studied, recent decades have seen an increased interest in understanding and controlling the dynamic, rheological properties of surfactant interfaces [4-7]. Surfactant interfaces are generally compressible, and thus exhibit a compressional modulus $G_{D}^{*}$ which may have a viscoelastic character, or equivalently a (complex) surface dilatational viscoelasticity $\eta_{D}^{*}$. This (possibly complex) surface dilatational modulus reflects surfactant processes that resist surface area changes, and thus gradients in surfactant concentration. Surface-active materials may also establish an (excess) surface shear viscosity $\eta_{s}^{*}$. In the continuum approximation, these surface-excess contributions to the stress are captured with a two-dimensional surface rheology that relates the surface-excess stress to the surface deformation. The Boussinesq-Scriven equations describe such interfaces in the continuum approximation, treating the surfactant layers as isotropic, homogeneous,

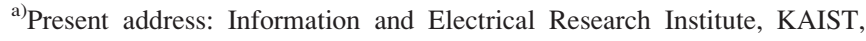
Daejeon 305-701, Korea

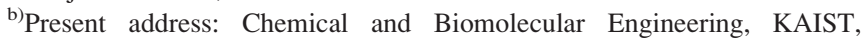
Daejeon 305-701, Korea

${ }^{c)}$ Author to whom correspondence should be addressed; electronic mail: squires@engineering.ucsb.edu
}

compressible Newtonian surface liquids, with surface dilatational $\left(\eta_{D}\right)$ and shear $\left(\eta_{s}\right)$ viscosities [8].

During the past century, various techniques have been developed for the measurement of surface shear rheological properties of surfactant interfaces. Classical canal surface viscometers were first developed to measure steady surface shear viscosities of surfactant monolayers flowing under surface tension gradients [9-12]. Indirect methods designed to interrogate purely shearing deformations were developed. They include rotating deep-channel viscometers [13-16] and rotating wall knife-edge viscometers [17,18], which externally impose some defined subphase flow, measure the resulting surface flow field using tracer particles, and deduce $\eta_{s}$ from hydrodynamic calculations.

A separate class of techniques for interfacial rheometry uses probes embedded within the interface, and externally forced or torqued to deform that interface. Surface rheological properties (e.g., $\eta_{s}^{*}$ ) are extracted from measured translational or rotational drag measurements. Various geometries have been used, including translating magnetic needles [e.g., the interfacial shear rheometer (ISR) [19-21], and rotating disks [22-24], biconical probes [25-27], Du Nouy rings $[28,29]$, and Double-Wall Rings (DWRs) [30]]. Active driving enables a broad range of interfaces to be measured, including linear and nonlinear rheological properties (e.g., yielding, shear thickening, or thinning), and time-dependent dynamics (e.g., aging).

A third class of technique for interfacial rheometry extends the core strategy of passive microrheometry-which relates the Brownian motion of micron-scale probes embedded within soft materials to their rheological properties [31-34] - to interfacial systems [35-44]. Small probe sizes naturally imply high sensitivity and measurement of local material properties. However, passive microrheology relies 
upon thermal fluctuations to drive the colloidal particles, and thus its practical use is generally limited to micro- or nanoscale probes, to materials with $\left|G^{*}\right|<1-10 \mathrm{~Pa}$ [45], and to surface with $\eta_{s}<1 \mu \mathrm{N} \mathrm{s} / \mathrm{m}$ [46].

Multiple qualitatively distinct modes may be excited as interfaces are deformed: In-plane modes include shear and dilatation, and out-of-plane modes include bending and torsion [47]. Deconvolving the contributions of each represents a nontrivial challenge for surface rheology. Indeed, many interfacial rheometry techniques generate deformations that couple at least two modes (e.g., shear and dilatational), requiring careful analysis - and possibly unsupported assumptions - to extract intrinsic material properties from experimental results. For example, interfacially adsorbed spheres and rods that are translated or rotated-either thermally or externally-introduce fore-aft compression expansion of the interface around the probe, exciting gradients in surfactant concentration $\Gamma$ and/or surface pressure $\Pi$. Such gradients increase the drag on probes translating within surfactant-laden interfaces (relative to the drag on a clean interface), even for interfaces with zero surface shear viscosity $\eta_{s}[48,49]$, by an $\mathrm{O}(1)$ amount for spheres and disks, and much greater for extended (rodlike) probes [50]. An additional complication includes possible translation-rotation couplings that would drive contact-line motion, which possesses a well-known singularity [51]. In this regard, surface deformations that are purely shear in character (e.g., established by rotating circular probes), or very nearly so (e.g., long, oscillating ISR needles), are advantageous for surface shear rheometry.

Another important challenge for interfacial rheometry arises from both the surface rheology $\left(\eta_{s}\right)$ of the surfactant monolayer and the bulk rheology $(\eta)$ of the underlying subphase. Such coupling makes it challenging to distinctly measure the rheological properties of the interface itself, as opposed to those of the bulk fluids. In particular, the interfacial drag is established by the interfacial stress $\left(\eta_{s} \nabla u\right)$ exerted along the perimeter of contact $\left(P_{c}\right)$ between the probe and interface layer, whereas the bulk drag arises due to the bulk viscous stress $(\eta \nabla u)$ exerted over the contact area $\left(A_{c}\right)$ between the probe and bulk phases. The dimensionless Boussinesq number $(B o)$

$$
B o=\frac{\eta_{s} P_{c}}{\eta A_{c}}
$$

gives the magnitude of the drag force exerted by the interface on a probe, relative to the drag force exerted by the bulk fluid(s) above and/or below the probe.

Measurements specifically sensitive to the surface shear rheological properties (e.g., $\eta_{s}, G_{s}^{*}$ ) should be performed in the $B o \gg 1$ (surface-dominated) limit as opposed to the $B o \ll 1$ limit (subphase-dominated). The Boussinesq number for a given system depends on both the rheological properties of the surface and bulk phases, as well as geometric factors, over which one has control. A characteristic surface viscosity scale,

$$
\eta_{s, \min }=\frac{\eta A_{c}}{P_{c}}
$$

emerges at $B o \approx 1$, a condition at which drag contributions from the subphase and the interface have the same order. This criterion gives a natural scale for the minimum surface viscosity that can be clearly distinguished from the subphase rheology. Notably, the perimeter to area ratio $\left(P_{c} / A_{c}\right)$ offers a geometric route to increase measurement sensitivity: Decreasing the probe size enables weaker surface shear moduli to be resolved. Passive microrheometry, for example, employs micron-scale colloids as probes for a high $\mathrm{s}$ $\left(\eta_{s, \min } \sim 10^{-3} \mu \mathrm{N} \mathrm{s} / \mathrm{m}\right)$.

To overcome some of these limitations, we have developed a technique for interfacial shear rheology that combines the sensitivity of microrheometry with the versatility, dynamic range, and control of macrorheometry. Furthermore, it affords additional advantages with interfacial visualization and welldefined viscometric deformations. In particular, we use electromagnets to externally torque microfabricated ferromagnetic button probes [52], whose orientation is tracked optically during their rotation. Frequency-dependent, linear viscoelastic, surface shear moduli can be measured by imposing smallamplitude oscillatory torques (stress), and measuring the resulting (oscillatory) displacements (strain). Nonlinear responses-e.g., strain hardening or softening, or yield stresses - may be probed by imposing large-amplitude oscillatory strains, steady torque (creep) or continuous rotation at a determined frequency. Unlike translating probes, the rotating microbutton can impose arbitrarily large strains, to give fully developed, Lagrangian-steady deformation fields.

As with traditional shear rheometry of bulk materials, where geometries are specifically designed to excite pure shear deformations, our rotating circular microbutton probe establishes a deformation field that is, in principle, pure shear, avoiding complications due to rheologically mixed flows that generally occur for non circularly symmetric probes and diffusing microrheometry particles. The interpretation of our measurements is thus more straightforward, as the hydrodynamic problem is relatively simple [49], avoiding contact-line motion, translation-rotation coupling, and compression/expansion of the surface.

While microbutton probes can be made with a range of sizes, we here focus on $10-50 \mu \mathrm{m}$ radii. Such dimensions give $P_{C} / A_{C}$ ratios (and therefore measurement sensitivities) that are $\sim 10^{2}-10^{3}$ higher than most macroscopic techniques. For example, $\eta_{s, \text { min }} \sim 4 \times 10^{-9} \mathrm{~N} \mathrm{~s} / \mathrm{m}$ for a $10 \mu \mathrm{m}$ microbutton on a water-air interface, and surface shear viscosities of this order can indeed be measured (e.g., Fig. 11). Moreover, small probes enable rheometry of relatively small sample volumes $(\sim \mu \mathrm{l})$ and surface areas $\left(\sim \mathrm{mm}^{2}\right)$.

Thus far, we have used microbutton probes to measure the interfacial rheology of phospholipid monolayers [53-56], colloidal monolayers [52,57], drying suspensions [58], and soluble surfactants [59]. In this article, we aim to describe in detail its design, operation, calibration, and capabilities. The article is constructed as follows: Sec. II deals with the design of the experimental apparatus. In Sec. III, we discuss the calibration methods and sources of experimental errors. Finally, we demonstrate the use of microbutton probes for surface rheometry in Sec. IV and bulk rheometry in Sec. V. 


\section{EXPERIMENTAL APPARATUS}

\section{A. Overview of technique}

A Janus ferromagnetic microbutton is deposited to a surfactant-laden fluid-fluid interface, where two pairs of orthogonally aligned electromagnets surrounding the surface are used to generate magnetic fields, thereby exerting desired torques or forces on the probe [Fig. 1(a)]. Figure 1(b) shows a general flow diagram for the experimental procedure. A data acquisition (DAQ) and function generator device controls the analog voltage output to a linear amplifier, which drives a current through the electromagnets surrounding the sample, generating a uniform magnetic field or magnetic field gradient within the area of interest. Microbuttons are visualized in bright field using a motorized zoom lens microscope (Navitar 6.5X) resting upon a motorized XYZ stage (ThorLabs). Images are acquired with a camera (JAI CVA10, 60 frames per sec) and frame grabber (NI PCI-1428). A custom LABVIEW code interfaced with the frame grabber and DAQ hardware simultaneously quantifies the microbutton position and angular orientation (via tracking the positions of two buttonholes on the probe) as well as the applied voltage to the electromagnets.

\section{B. Microbutton probes}

Our active, interfacial microrheometry technique employs probes that are (i) small, yet visible under optical microscopy; (ii) ferromagnetic, to enable torques or forces to be externally applied; (iii) amphiphilic, to ensure the probes physically adsorb onto fluid-fluid interfaces; and (iv) orientationally anisotropic and thus rotationally trackable. To satisfy these requirements, we synthesize microbuttons with radius generally equal to 10 or $50 \mu \mathrm{m}$ using twodimensional, layer-by-layer photolithographic techniques $[60,61]$, enabling multiple functionalities to be incorporated onto any initial shape with each added layer. Choi et al. [52] described the detailed fabrication procedure. Briefly, a sacrificial layer (Omnicoat, Microchem) and photoresist layer
(SU-8, Microchem) with 2 and $10 \mu \mathrm{m}$ thickness for 10 and $50 \mu \mathrm{m}$ buttons, respectively, are spin-coated onto a silicon wafer, then exposed to ultraviolet (UV) light through a patterned photomask to crosslink the photoresist in the desired microbutton shape. Thin layers of nickel (typically $150 \mathrm{~nm}$ ) and gold (typically $10 \mathrm{~nm}$ ) are added via electron beam physical vapor deposition. The nickel layer imparts an in-plane ferromagnetic moment, and the gold layer enables Janus amphiphilicity by adding self-assembled thiol monolayers, typically fluorocarbon-thiol or alkane-thiol. This ferromagnetic layer is magnetized with a strong, permanent magnet held close to the wafer, before the disks are released from the wafer.

\section{Langmuir trough and sample holder}

Langmuir troughs provide an excellent experimental platform for the study of equilibrium and dynamic rheological properties of insoluble surfactant monolayers, as the surface area (and thus surfactant surface concentration of a fixed amount of molecules spread to the surface) can be easily be controlled with a movable barrier. We have developed multiple systems [e.g., Fig. 2(a)], wherein a stepper motor (PK245-01AA, Oriental Motor) and motor controller(s) (VXM, Velmex) controls motion of one or two barriers (either Teflon or Delrin) within a Teflon trough (area $170 \mathrm{~cm}^{2}$ ), enabling uniaxial compression of the surface from one side or two sides, at rates between 0.9 and $45 \mathrm{~cm}^{2} / \mathrm{min}$. The surface pressure $(\Pi)$ is measured using a Wilhelmy plate tensiometer (Reigler \& Kirstein) equipped with a filter paper plate. The relatively large surface area and subphase depth $(\sim 1-2 \mathrm{~cm})$ in our trough system, however, can lead to large surface convection due to either Marangoni flows or subphase fluid motion due to barrier translation. In many cases, it is necessary to minimize such convection, to ensure microbuttons remain within the field of view and that the interface is stable. We thus employ a custom-designed sample holder [Fig. 2(b)], made of aluminum or delrin, which can be inserted into the Langmuir trough, and which houses both the electromagnets and a small aluminum cone insert at
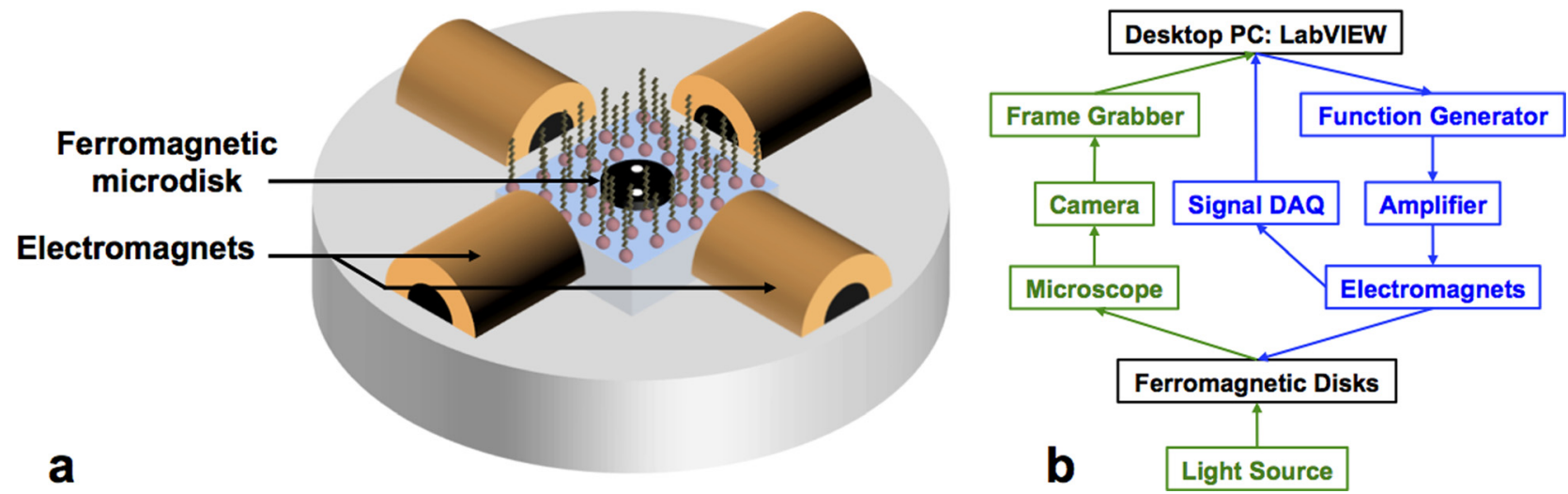

FIG. 1. (a) Microbutton microrheometry schematic: Two pairs of orthogonally aligned electromagnets generate desired magnetic fields or magnetic field gradients and thus torques or forces on a Janus ferromagnetic microbutton placed within a surfactant layer. (b) Experimental flow chart: A LABVIEW program on a desktop computer interfaced with a function generator controls the voltage output to a linear amplifier. The amplified signal, monitored with DAQ equipment interfaced with the desktop PC, drives an electric current $(\leq 1 \mathrm{~A})$ through electromagnet coils. The resulting magnetic field exerts a desired torque or force on a ferromagnetic microbutton probe placed at a fluid-fluid interface, exerting a shear stress. A charge-coupled device (CCD) camera and frame grabber interfaced with the Desktop computer capture a bright-field image of the microbutton through a microscope, enabling the (realtime) tracking of the two buttonholes, and thus the rotational displacement. 


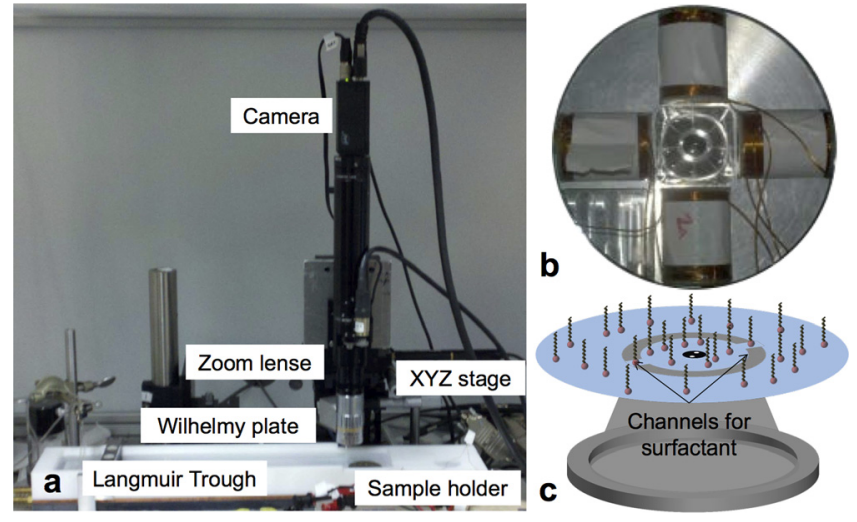

FIG. 2. (a) The experimental setup for microbutton surface rheometry consists of a sample/electromagnetic holder placed within a Langmuir trough, a Wilhelmy plate for surface pressure measurements, and bright-field microscopy using a zoom lens and CCD camera. (b) An aluminum sample holder for the two pairs of orthogonally aligned electromagnets to generate a desired magnetic fields or magnetic field gradients in any direction in the plane. (c) Schematic of aluminum cone insert: Two thin side slits suppress stray convective flows and allow surfactants to freely move in and out of the cone along the air-water interface, which is pinned at cone edges. Reprinted with permission from Zell et al., Proc. Natl. Acad. Sci. U.S.A. 111(10), 3677-3682 (2014). Copyright 2014, PNAS [59].

the center [Fig. 2(c)]. Upon adding liquid subphase to the trough, the liquid-air interface becomes pinned at the $5 \mathrm{~mm}$ diameter rim of the cone, thus creating a small planar liquid surface within which probes are deposited, and where the magnetic field has been calibrated. A thin channel $(\sim 500 \mu \mathrm{m}$ wide) cut into walls of the cone rim allow surfactant to flow in and out of the center, with the walls minimizing surface disturbances and convective flows $[62,63]$. The internal sample holder area is itself isolated from the larger reservoir of the trough by a narrow 1-mm-wide channel. Moreover, disturbances to the interface are minimized by mounting the entire setup on a vibration isolation engineering table (Newport), within a custom-designed cabinet to reduce air currents and dust. Additionally, the Langmuir trough remains fixed in place on the table, while the microscope position is controlled with a motorized stage. Finally, the sample holder can also function as a standalone chamber for bulk materials or soluble Gibbs monolayers, whose surface pressure, surface concentration, and dynamics are controlled by the surfactant concentration in the bulk.

\section{Modes of active microrheometry}

Distinct measurement modes-oscillatory stress, creep, steady rotation, and translation-can be performed with active microbutton rheometry, by direct analogy to macroscopic rheometry. As with conventional rheometry, each is suited to a particular measurement.

The oscillatory rotational stress mode is used to measure the frequency-dependent linear viscoelastic shear moduli of a bulk material $\left(G^{*}(\omega)\right)$ or a surface $\left(G_{s}^{*}(\omega)\right)$ by driving rotational motion of the probe with an externally applied magnetic torque. A low-amplitude voltage signal from the function generator is passed through the amplifier input. The output from the amplifier drives a current through an aligned pair of electromagnets, establishing a relatively uniform magnetic field $\vec{B}$ (with orientation $\theta_{B}$ ) within the area of interest. The magnetic field is applied perpendicular to the orientation $\theta_{m}$ of the magnetic moment $\vec{m}$ of the microbutton (so that $\left|\theta_{B}-\theta_{m}\right|=\pi / 2 \pm 0.2$ radians). In so doing, the coils exert a torque on the ferromagnetic probe, $\vec{L}=\vec{m} \wedge \vec{B}$, with magnitude $|\vec{L}|=m B \sin \left(\theta_{B}-\theta_{m}\right)$ that is well-approximated by $L_{0} \sim m B$, with errors of order $\left(\pi / 2-\left|\theta_{B}-\theta_{m}\right|\right)^{2}$.

An oscillatory torque $\left(L_{0} e^{i \omega t}\right)$ is applied to the microbutton by imposing an oscillatory magnetic field $B_{0} e^{i \omega t}$ at a particular frequency $(f=0.1-100 \mathrm{~Hz})$,

$$
L_{0} e^{i \omega t}=m B_{0} e^{i \omega t},
$$

while bright-field microscopy is used to record the resulting angular displacement of the microbutton,

$$
\theta_{m}(t)=\theta_{m, 0}+\Delta \theta_{0}^{*} e^{i(\omega t)},
$$

where $\Delta \theta_{0}^{*}$ is a complex strain amplitude, which can be expressed explicitly in terms of a magnitude and phase lag via

$$
\Delta \theta_{0}^{*}=\Delta \theta_{0} e^{-i \omega \delta}
$$

Under oscillatory torquing at frequency $\omega$, the angular displacement $\theta_{m}$ obeys a torque balance

$$
\left(-\omega^{2} I_{R}^{*}+i \omega \zeta_{R}^{*}+k_{R}^{*}\right) \Delta \theta_{0}^{*}=L_{0},
$$

where $I_{R}, \zeta_{R}$, and $k_{R}$ represent the (possibly complex and frequency dependent) moment of inertia, rotational viscous resistance, and rotational spring constant of the microbutton within the interface/subphase system. At frequencies $\omega$ that are sufficiently low to neglect inertia may be neglected (under conditions specified below), the torque balance reduces to

$$
\left(i \omega \zeta_{R}+k_{R}\right) \Delta \theta_{0}^{*}=m B_{0} .
$$

The frequency-dependent rotational resistance,

$$
\zeta_{R}^{*}(\omega)=\zeta_{R}+\frac{k_{R}}{i \omega},
$$

is then obtained via

$$
\zeta_{R}^{*}(\omega) \approx \frac{m B_{0}}{i \omega \Delta \theta_{0}^{*}} .
$$

Controlled strain measurements can be performed by forcing the microbutton to rotate at a given angular velocity using a strong magnetic field $B_{0}$ that rotates with angle $\theta_{B}(t)=\Omega t$. Measuring the orientation $\theta_{m}(t)$ of the magnetic moment, then, gives the angular lag $\Delta \theta$ between the applied field $\theta_{B}(t)$ and the magnetic moment $\theta_{m}(t)$. The steady rotational resistance is then given by $\zeta_{R}(\Omega)=\left(m B_{0} \sin \Delta \theta\right) / \Omega$.

Rotational creep measurements can be performed by tracking the microbutton orientation $\theta_{m}(t)$ in real time, and rotating 
the direction $\theta_{B}(t)$ of the applied field (but maintaining a constant field strength $\left.B_{0}\right)$ according to $\theta_{B}(t)=\theta_{m}(t)+\pi / 2$, to impose a steady applied torque $\left|L_{0}\right|=m B_{0}$.

Difficulties in measuring the angle between the applied field and the magnetic moment $\Delta \theta$ render these two methods to be less precise than oscillatory stress, and a more complex continuum-mechanical problem must be solved to interpret measurement in terms of intrinsic surface rheological properties. However, they enable nonlinear rheological measurements and both methods have been used to measure the surface yield stress of a liquid condensed phase of a monolayer of the phospholipid dipalmitoylphosphatidylcholine (DPPC) [54,55].

Finally, the probe can be forced to translate by applying an external magnetic force, rather than torque. All of the rotational measurements described above employ a spatially homogeneous magnetic field, imposed using electromagnet pairs connected in series along a single circuit. Translational measurements, by contrast, require magnetic field strength gradients, which are obtained by controlling each electromagnet individually. Driving a current through a single electromagnet's coil establishes a positiondependent B-field (and therefore B-field gradient) that exerts a magnetic force $\left(\mathbf{F}_{\text {mag }}=\mathbf{m} \cdot \nabla \mathbf{B}\right)$ on the microbutton. The resulting translation velocity $U$ is then measured by tracking the microbutton position in time, which yields a translational drag coefficient $\zeta_{T}$

$$
\zeta_{T}=\frac{m|\nabla B|}{|U|} .
$$

\section{Analysis and interpretation}

The rotational resistance $\zeta_{R}$ or translational resistance $\zeta_{T}$ are not properties intrinsic to the material itself, but depend on the size and shape of the probe, and how it is forced. In order to extract intrinsic material properties (e.g., $\eta_{s}, \eta_{D}, G_{s}^{*}(\omega)$, and so on), a continuum-mechanical problem must be solved. As in macroscopic rheometry, such computations become much more difficult—and at times essentially impossible-if the flow field is not viscometric (e.g., pure shear for shear viscosity). In this regard, rotating circular microbuttons hold a particular advantage as microrheological probes, as they establish a well-defined viscometric flow field, with shearing deformations that do not extend or dilate/compress the surface.

The rotational resistance $\zeta_{R}$ of a thin, circular disk of radius $a$ rotating quasisteadily within a viscous monolayer above a liquid subphase has been computed [49] and depends on both the surface shear viscosity $\left(\eta_{s}\right)$ and the subphase viscosity $(\eta)$, whose relative importance is given by the Boussinesq number [Eq. (1)], here defined as

$$
B o=\frac{\eta_{s}}{\eta a} \text {. }
$$

Measurements specifically sensitive to $\eta_{s}$ should be performed in the $B o \gg 1$ (surface-dominated) limit, in which case the surface viscosity is given by

$$
\eta_{s}=\frac{\zeta_{R}}{4 \pi a^{2}}
$$

More generally, the $B o \gg 1$ limit of the (complex) surface shear modulus for viscoelastic interfaces is given by

$$
G_{s}^{*}=\frac{i \omega \zeta_{R}^{*}}{4 \pi a^{2}} .
$$

In the opposite (subphase-dominated) limit $(B o \ll 1)$, the subphase viscosity can be determined from the rotational resistance according to

$$
\eta=\frac{3 \zeta_{R}}{16 a^{3}},
$$

and the viscoelastic moduli for complex subphase fluids is given by

$$
G^{*}=\frac{3 i \omega \zeta_{R}}{16 a^{3}}
$$

in the $|B o| \ll 1$ limit.

Equations (12) and (14) assume the probe and fluid motions to be quasisteady, the validity of which depends upon the relative importance of the (unsteady) inertia of the subphase. (We generally assume that the excess inertia associated with the two-dimensional interface is negligibly small.) Defining a Reynolds number for the transient inertia via $R e=\rho \omega a^{2} / \eta$ [21] reveals unsteady subphase inertia to be negligible whenever $R e \ll 1$, corresponding to a frequency limited to

$$
f \ll f_{I}=\frac{\eta}{2 \pi \rho a^{2}} .
$$

This inertial frequency $f_{I}$ is $10^{3} \mathrm{~Hz}$ and $40 \mathrm{~Hz}$ for $10 \mu \mathrm{m}$ - and a $50 \mu \mathrm{m}$-radius microbuttons, respectively, both of which exceed the working range of our experiments (typically $0.1-10 \mathrm{~Hz}$ ). Once probes are driven at or near the inertial frequency, the oscillatory boundary layers driven within the subphase flow become comparable to the probe radius, and thus modify the scaling argument used to estimate the Boussinesq number. Care must thus be taken with larger probes near this inertial frequency, particularly around $B o \sim 1$, as is known for the ISR [21].

By contrast, the translational motion of probes (e.g., spheres, disks, or rods) or the rotational motion of nonaxisymmetric probes (e.g., rods) along a surfactant monolayer drive rheologically mixed flows that deform the surface with shear, extension, and dilation. Since $\zeta_{T}$ combines the effects of shear and dilation into one measured quantity, it is not generally possible to quantitatively extract $\eta_{s}$ from $\zeta_{T}$ measurements alone.

Theoretical treatments of translational motion typically decouple the hydrodynamic problem from the surfactant mass transport at the surface by making assumptions about the dilatational mechanical properties within the monolayer. For example, Danov et al. [64] assumed a constant surface pressure in solving for the translational resistance 
of a sphere moving within a monolayer, considering excess dissipation due to constant surface shear and dilatational viscosities. The opposite extreme of effectively incompressible 2D interfaces introduces surface pressure gradients (and therefore Marangoni stresses) as well, whereupon the translational resistance $\zeta_{T}$ becomes independent of dilatational viscosity $\eta_{D}$, yet not unambiguously relatable to $\eta_{s}$ $[48,49,65,66]$.

In particular, the translational resistance of a thin, circular disk translating along a surfactant-free liquid surface is given by [67]

$$
\zeta_{T, \text { clean }}=\frac{16}{3} \eta a
$$

When an incompressible surfactant is present, however, the translational resistance changes qualitatively, even if that surfactant has zero surface-shear viscosity. Enforcing 2D surface incompressibility fundamentally changes the boundary condition imposed on the subphase flow, which qualitatively changes the subphase flow over an $\mathcal{O}(a)$ region, modifying $\zeta_{T}$ even in the $B o \ll 1$ limit [49]

$$
\zeta_{T}(B o \ll 1)=8 \eta a .
$$

The incompressibility condition acts to increase the relative translational resistance $\zeta_{T} / \zeta_{T \text {, clean }}$ by $3 / 2$ in the low-Bo limit, irrespective of the surface shear viscosity of the monolayer. Fischer et al. $[48,68]$ highlighted this point in discussing the difficulty of extracting surface shear viscosities from translating probes in the low-Bo limit.

Another complication arises with soluble surfactants, where finite kinetics of adsorption/desorption from compressing/dilating surfaces introduces additional dissipative processes, acting like an effective surface-excess viscosity due to dilatational deformations [69-71]. It is thus generally difficult to extract shear and/or dilatational viscosity from measured increases in translational drag when $\zeta_{T} / \zeta_{T \text {, clean }}$ is $\mathcal{O}(1)$.

Only when the translational resistance greatly exceeds subphase-dominated values of $\mathcal{O}(\eta a)$ can $\eta_{s}$ be reliably quantified. In the large-Bo limit, the translational resistance $\zeta_{T}$ of a thin disk is given by [49]

$$
\zeta_{T}(B o \gg 1)=\frac{4 \pi \eta_{s}}{\ln (2 B o)-\gamma_{E}+\frac{2}{B o \sqrt{\pi}}-\frac{\ln (2 B o)}{2 B o^{2}}},
$$

where $\gamma_{E}$ is Euler's constant.

Rotational measurements hold several other advantages over translation. For instance, arbitrarily large strains can be applied, and fully developed, Lagrangian-steady deformation fields can be established, with steadily rotating probes. For a given magnetic field, a larger probe velocity and effective shear rate can be obtained for rotation than translation. In the $B o \gg 1$ limit, where $\zeta_{R}$ is given by Eq. (12), the azimuthal velocity around a rotating microbutton scales like

$$
v_{R} \sim \frac{m B}{\eta_{s} a}
$$

whereas the velocity around a translating probe scales like

$$
v_{T} \sim \frac{|\mathbf{m} \cdot \nabla \mathbf{B}|}{\eta_{s}} .
$$

Since the magnetic field gradient strength in our apparatus scales like $|\nabla B| \sim B_{0} / L$, where $L$ is the $\mathrm{O}(\mathrm{cm})$ length scale between the electromagnets, probe velocities under rotational forcing exceed those under translational forcing by a factor

$$
\frac{v_{R}}{v_{T}} \sim \frac{B_{0}}{|\nabla B| a} \sim \frac{L}{a}
$$

On the other hand, the rotational resistance of circular probes depends strongly on the local rheology of the interface around the boundary of the probe, and is exceedingly sensitive to slip between the probe and the interface. Probe translation-which is much less sensitive to interfacial slip — can thus complement $\zeta_{R}$ measurements, by providing a valuable control against slip.

\section{E. Tracking microbutton orientation}

In order to measure rotational or translational drag coefficients, the angular orientation and position of the microbutton needs to be accurately and reliably quantified. To determine its orientation in real time from bright-field images of the microbutton, a LABVIEW program tracks the centroids of the buttonholes, adapting the strategy that Crocker and Grier [72] introduced for colloidal particle tracking. Figure 3 illustrates the tracking algorithm used to find the angular orientation that begins with acquisition of a bright-field digital image $(760 \times 560$ pixels $)$ of the microbutton $(a>50$ pixels). Each pixel location has an intensity value between 0 and 255, and we determine the location of the darkest pixel, assumed to be located on the microbutton [Fig. 3(a)]. An image of width $4 \mathrm{a}$, centered at the darkest pixel, is then cropped from the original image to restrict the search area. To find the center of the disk, a black square of width $\sqrt{2 a}$ is convoluted with the cropped image in Fig. 3(b), where the maximum of this convolution represents the disk center.

We have developed two different methods to determine the location of the buttonholes, and thus the angular orientation from the cropped disk image [Fig. 3(c)]. The first method is a tracking algorithm in LABVIEW. It is fast enough, even with a basic computer, for real-time tracking. The second method uses built-in image processing commands within MATLAB, and is more accurate but slower.

In the first method, a white square (of width $\sqrt{2}$ times the buttonhole radius) is convoluted with the cropped disk image [Fig. 3(d)], the maximum of which represents the centroid of the first buttonhole. To locate the other buttonhole, we black out the first buttonhole identified with a black square, and repeat the process.

In the second method, the buttonhole locations on the cropped disk image are determined by a MATLAB script using 

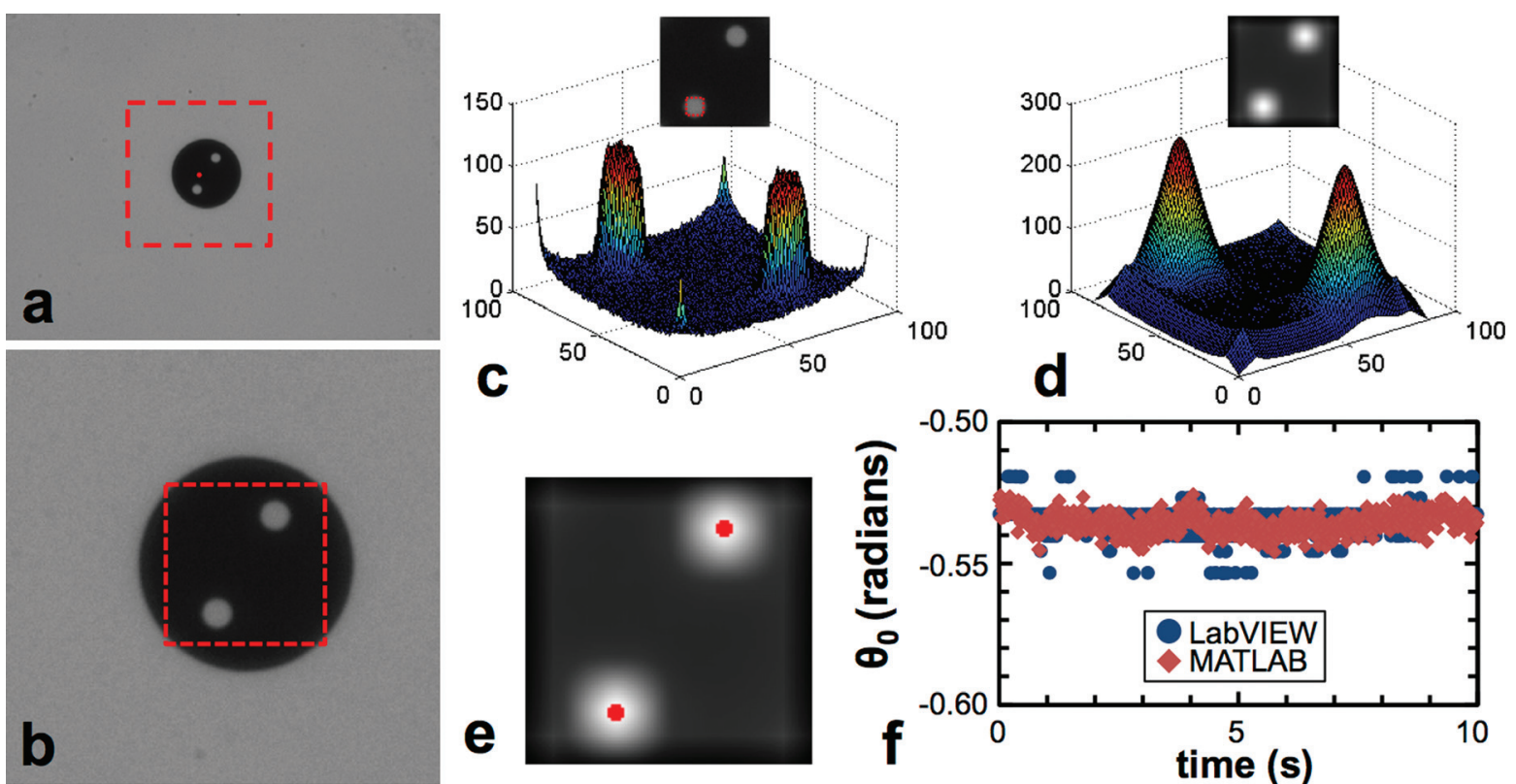

FIG. 3. A LABVIEw program tracks the position of two buttonholes on the microbutton in realtime. (a) The original bright-field image, which is cropped with a square centered on the darkest pixel. (b) A black square (dashed) is convoluted with the cropped image to locate the center of the microbutton. (c) The center of the microbutton found from (b). To find the buttonhole locations, a white square is convoluted with the cropped disk image in (c), giving the convoluted image in (d). The locations of the maximum for each buttonhole are indicated in the convoluted image in (e). (f) The orientation of a microbutton at the airwater interface is tracked for $10 \mathrm{~s}$ using the LABVIEW and MATLAB-based algorithms, revealing standard deviations of 0.005 and 0.003 rad, respectively.

the circular Hough transform function imfindcircles within the LABVIEW code.

The angular orientation is determined from the relative buttonhole locations [Fig. 3(e)] and is given by either $\theta=$ $\tan ^{-1}(\delta x / \delta y)$ or $\theta=\tan ^{-1}(\delta y / \delta x)$. Figure 3(f) plots the angular orientation of a single, stationary microbutton resting on an air-water interface for $10 \mathrm{~s}$. The standard deviations of the angle for the LABVIEW and the MATLAB methods are found equal to 0.005 and $0.003 \mathrm{rad}$, respectively.

\section{F. Electromagnets}

Custom-designed electromagnets are used to control the torques and forces exerted on the ferromagnetic microbutton probes. Each electromagnet uses 28 AWG, polymer-coated copper wire (Belden 8080) wrapped around a plastic spool, with electrical resistance of approximately $3.1 \Omega$. The spool allows a $6.3 \mathrm{~mm}$ diameter iron rod (purity $>99 \%$ ) to be inserted concentrically into the electromagnet center, amplifying the magnetic field by a factor of about 4 . Two sets of electromagnet pairs, aligned in the sample holder on two orthogonal axes 1 and 2, are used to generate magnetic fields of specified magnitude in any planar direction. Electromagnets on axis 1, which are connected in series and controlled from a single output, are used to generate time dependent, but spatially uniform, magnetic fields (e.g., $B_{0} e^{i \omega t} \hat{\mathbf{e}}_{1}$ in oscillatory mode). The two electromagnets on axis 2 are controlled independently, enabling the generation of magnetic field gradients along the 2 axis required for translation mode. They can also establish a uniform magnetic field when controlled simultaneously to a) initially align the probe perpendicular to axis 1 in oscillatory mode, or b) drive rotation in creep or controlled rotation modes. A DAQ and function generator device (NI PCIe-6353) controls the ana$\log$ voltage output to a linear amplifier (axis 1: Sony STRDH100, axis 2: Rheomics, RTA), which drives a current through the electromagnets contained in the sample holder. The voltage across each set of electromagnets is recorded with the analog inputs of the same DAQ device.

\section{CALIBRATIONS}

\section{A. Magnetic field}

The ferromagnetic probe is manipulated using a magnetic field imposed by two pairs of electromagnets. Applying a current through a single electromagnet [Fig. 4(a)] creates a magnetic field that decreases with distance from the end of the coil [Fig. 4(b)]. When current is run through two aligned coils [e.g., pair 1a/1b in Fig. 4(a)] in the same sense, the resulting field is uniform to within $\pm 2.5 \%$ in a $1 \mathrm{~mm}$ region in the center of the two magnets. This magnetic field is used to exert a torque on the microbutton, and thus to force it into rotation.

The relationship between electric current and magnetic field is measured to be linear for all currents up to $\pm 1 \mathrm{~A}$ [Fig. 4(c)]. No higher current can be imposed without damaging the coils. The presence of iron cores inside the coils increases the magnetic field four-fold, and enables higher magnetic field strengths $\left(B_{\max }=120 \mathrm{G}\right)$ to be imposed, as would be required to measure high viscosities. These measured relations are used to relate the imposed magnetic field (and therefore the torque imposed on the microbutton) to the applied current during experiments.

Forcing probes to translate along axis 2 requires a magnetic field gradient [Fig. 4(d)] imposed by magnets $2 \mathrm{a} / 2 \mathrm{~b}$, 

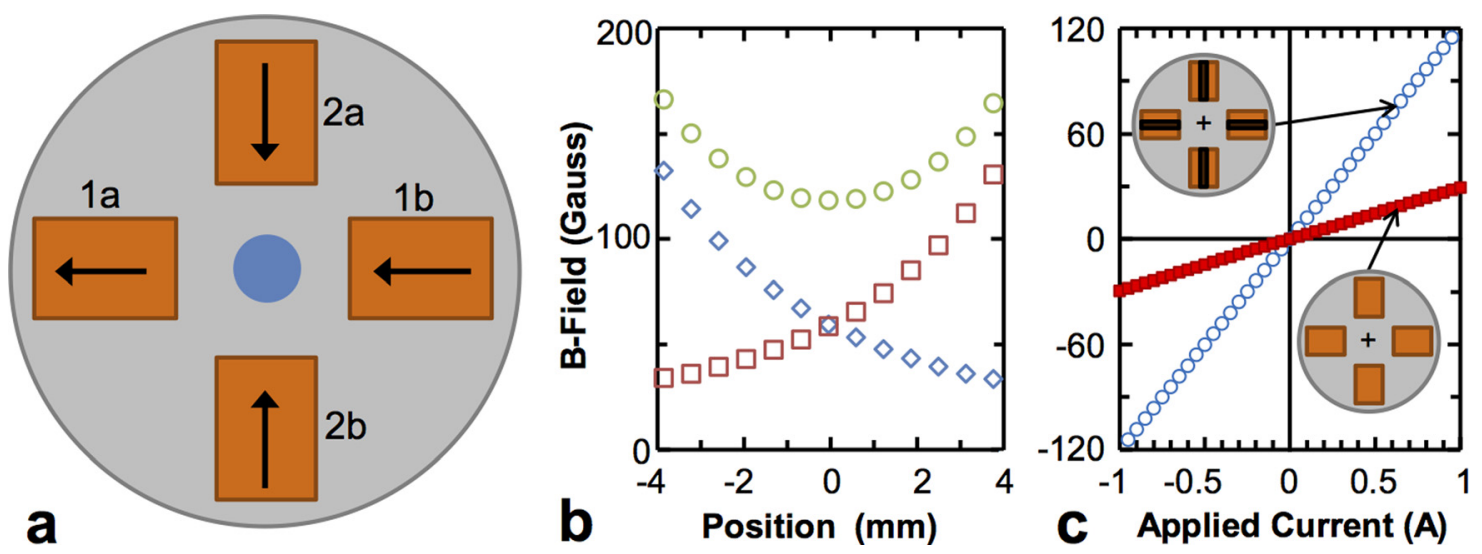

FIG. 4. (a) Schematic drawing of magnetic field axes 1 and 2. Coils 1 a and $1 \mathrm{~b}$ generate fields in the same direction, creating relatively homogeneous fields, whereas coils $2 \mathrm{a}$ and $2 \mathrm{~b}$ generate fields in opposing directions to create relatively homogeneous gradients. (b) Magnetic field strength vs. position along axis 1 at $1 \mathrm{~A}$, with Fe cores inserted, for three different configurations: $(\bigcirc)$ 1a $+1 \mathrm{~b} ;(\diamond) 1 \mathrm{a} ;(\square) 1 \mathrm{~b}$. (c) Magnetic field amplitude, measured at the sample center with (O) and without ( $\mathbf{\square})$ Fe core inserts, grows linearly with the direct current (DC) amplitude. (d) Magnetic field strength gradient along the electromagnet axis versus distance from the sample center, produced for 1 A current through coil $1 \mathrm{a}(\diamond) ; 1 \mathrm{~b}(\square)$; and $2 \mathrm{a}+2 \mathrm{~b}$ simultaneously $(\bigcirc)$. Field strength and gradient strengths vary by less than $15 \%$ within the $1.5 \mathrm{~mm}$ from the center, where experiments are performed.

for which two methods can be used. First, a homogeneous gradient can be imposed by running oppositely directed currents through each electromagnet in a pair. The oppositely directed magnetic fields give raise to a magnetic field gradient that is constant to within 5\% variations in the $1 \mathrm{~mm}$ experimental region centered between the electromagnets. In the second approach, currents are run through individual magnets sequentially: Establishing a field (and gradient) with magnet $2 \mathrm{a}$ alone causes probe translation toward magnet $2 \mathrm{a}$; running the current through electromagnet $2 \mathrm{~b}$ pulls the probe in the opposite direction. While the two methods give equivalent results - the force depends linearly on the magnetic gradient-the second method allows multiple repeated measurements, as probes can be pulled back and forth (without $180^{\circ}$ microbutton rotations that might destroy local interfacial microstructure).

Having determined the relationship between the static magnetic field and applied current, we now determine the response time of the electromagnets. Electromagnet coils
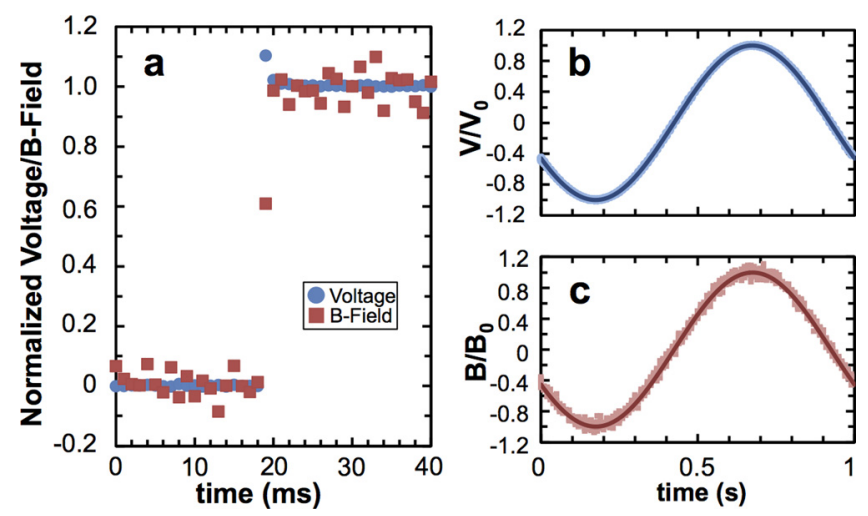

FIG. 5. (a) Response of the magnetic field ( $\square$ ) following a step increase in voltage (1) through the coils. The magnetic field adjusts to a steady value on the order of $1 \mathrm{~ms}$. (b) and (c) Imposing an oscillatory potential (b) across the electromagnetic coils at $1 \mathrm{~Hz}$ gives a $1 \mathrm{~Hz}$ sinusoidal magnetic field (c) measured with a Hall probe. The black lines correspond to a sinusoidal fit. The time lag between the current and the magnetic field is $1.2 \mathrm{~ms}$, consistent with the step-change experiment (a). behave as Inductor-Resistor (LR) circuits, where inductance $L$ is $2 \mathrm{mH}$ when iron cores are inserted, and $0.65 \mathrm{mH}$ without, for a response time $L / R=0.6 \mathrm{~ms}$ with cores, $0.2 \mathrm{~ms}$ without. Figure 5(a) shows the magnetic field, measured with a Hall probe (F.W. Bell, 5180 Gauss Meter), compared with voltage across the electromagnet pair, following a step change in applied current. The B-field adjusts to its static value approximately $1 \mathrm{~ms}$ after the step change. To confirm this response time, we measure the B-field response due to a sinusoidally applied current at $1 \mathrm{~Hz}$ [Figs. 5(b) and 5(c)]. Fitting the results of each to a sine waveform reveal a time lag between the current and magnetic field of $1.2 \mathrm{~ms}$. These three results are consistent, confirming that magnetic fields can be reliably imposed at frequencies below $\sim 100 \mathrm{~Hz}$.

\section{B. Synchronization of microbutton orientation and applied torque}

In measuring the linear viscoelastic moduli under oscillatory stress, it is crucial to accurately measure the phase lag between the stress and strain [73]. Even small errors in phase lag $\delta$ provide errant results, particularly when $\delta$ approaches either zero or $\pi / 2$, corresponding to the limits of purely elastic or purely viscous materials, respectively. Although the voltage has been shown to be synchronized with the established magnetic field to within $\sim 1 \mathrm{~ms}$ as described above, we must also synchronize video microscopy with the applied voltage. To do this, we connect a light emitting diode (LED) indicator (NTE Electronics, Inc., NTE30037) in series with the electromagnet coils. Since the LED has a very short response time $(\sim 250 \mathrm{~ns})$, the emitted light is in phase with the electric current. With the LED positioned directly under the camera, we measure the average intensity of the image collected by the camera due to the emitted light as well as the voltage across the electromagnet-LED circuit measured with the DAQ hardware as a function of time. Figure 6 shows the normalized voltage and light intensity response as function of time. We fit the voltage with a sinusoidal function, and the light intensity with a Gaussian function. The 


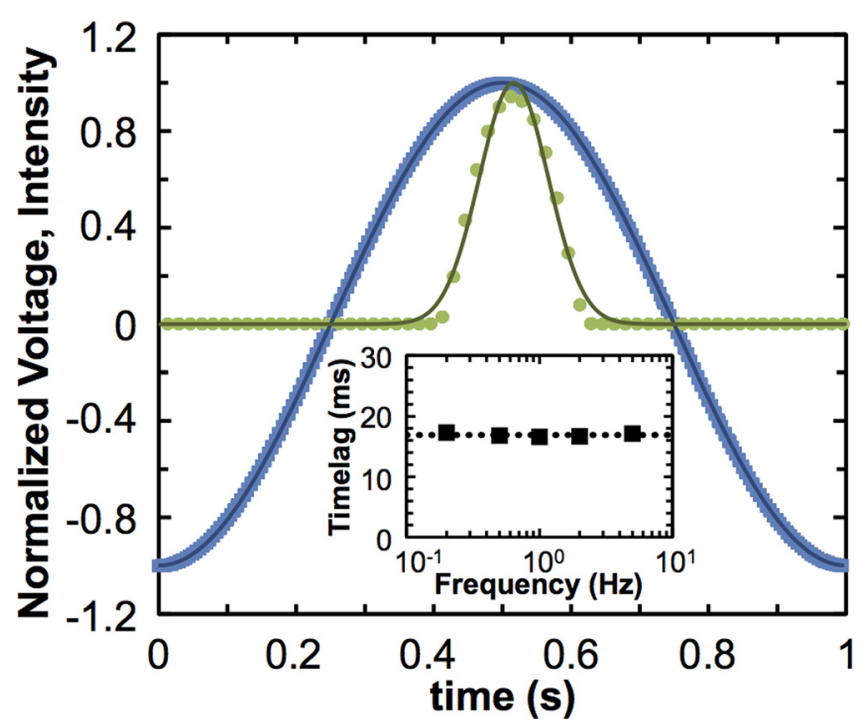

FIG. 6. LED light intensity is measured by applying $1 \mathrm{~Hz}$ oscillatory voltage across electromagnets as a function of time. The measured voltage signal is fit with a sinusoidal function, while the light intensity is fit with a Gaussian function. The peak-to-peak time difference gives the time lag between video microscopy and electric current. Inset: The time lag is equal to $16.9 \mathrm{~ms}$ and independent of frequency.

light intensity is found to lag the voltage by $16.9 \pm 0.4 \mathrm{~ms}$, independent of the frequency, which appears related to the time between images captured $(1 / 60 \mathrm{~s})$. Since different cameras and image acquisition systems may give different time lags, this synchronization step is important in ensuring the phase angle is measured precisely. With the present system, the phase lag can be measured with $0.4 \mathrm{~ms}$ precision.

\section{Magnetic moment of microbutton probes}

The in-plane magnetic moment $(m)$ of the microbutton is required to determine the torque applied to the microbuttons. Since it is often not practical (or sometimes possible) to measure the magnetic moment of a particular microbutton before using it for a measurement, we here measure the magnetic moment of multiple microbuttons from various fabrication batches, both to determine the variance of $m$ within a particular set of nominally identical microbuttons, and also to determine how the average $m$ depends on the microbutton radius and nickel layer thickness.

To measure the magnetic moment of individual probes, we first deposit a microbutton onto the surface of a purely viscous liquid of known (or measurable) viscosity (typically water). We perform a dynamic frequency sweep measurement of the viscous component of the rotational drag coefficient $\zeta_{R}^{\prime}(\omega)$ using Eq. (9), where the only unknown quantity is $m$. Using the thin-disk limit of the rotational drag coefficient due to an underlying viscous subphase [Eq. (14)], we solve for the magnetic moment of the microbutton

$$
m=\frac{16 \eta a^{3} \omega \Delta \theta_{0}}{3 B_{0} \sin \delta}
$$

Histograms in Fig. 7 for microbuttons with radii of 10 and $50 \mu \mathrm{m}$, each with $\mathrm{Ni}$ layer thicknesses of $150 \mathrm{~nm}$, reveal magnetic moments of $(52 \pm 8) \times 10^{-17}$ and $(970 \pm 200) \times$ $10^{-17} \mathrm{~J} / \mathrm{G}$, respectively.

Notably, these distributions are only obtained when the ferromagnetic layer of the microbuttons are saturated using a strong external magnet before release from the wafer. Without this step, the magnetic moment distribution has a smaller mean, and a broader distribution.

A complementary measurement of the microbutton magnetic moment can be performed using translational measurements. A magnetic field strength gradient is imposed along the axis 2 in order to exert a translational drag force on the microbutton. The microbutton is forced toward one electromagnet by applying a current through that electromagnet's coil, then in the opposite direction with a current through the other electromagnet's coil, keeping the microbutton within a $500 \mu \mathrm{m}$ location. The translation velocity is averaged over forward and reverse directions, giving
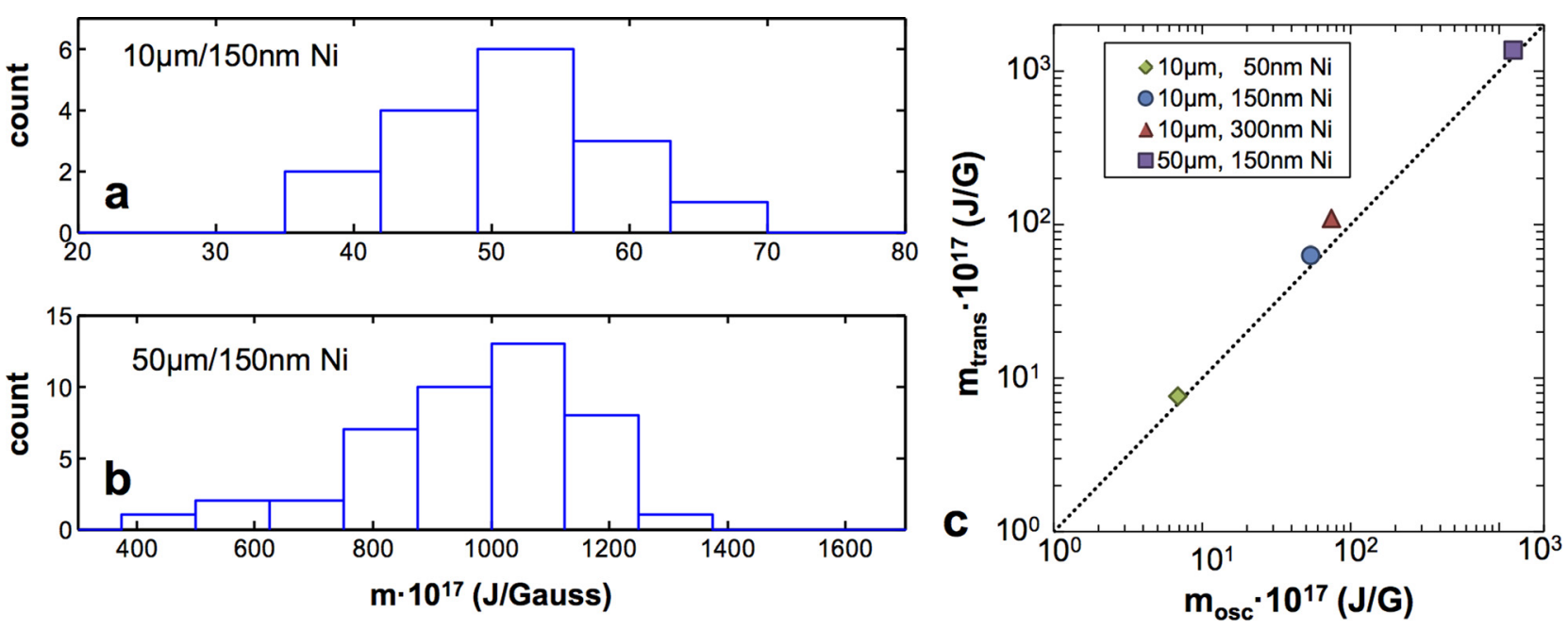

FIG. 7. Histogram distribution of the magnetic moment measured by rotation for microbutton types of (a) $10 \mu \mathrm{m} / 150 \mathrm{~nm}$ Ni and (b) $50 \mu \mathrm{m} / 150 \mathrm{~nm}$ Ni determined from calibration measurements at the air-water interface. (c) Magnetic moment extracted from translational measurements plotted against magnetic moments measured via oscillation for different microbutton types. A good agreement between the two measurements methods is observed. Reprinted with permission from Zell et al., Proc. Natl. Acad. Sci. U.S.A. 111(10), 3677-3682 (2014). Copyright 2014, PNAS [59]. 


$$
\langle|U|\rangle=\frac{m\langle|\nabla B|\rangle}{\zeta_{T}}
$$

Using the thin-disk limit of the translational drag coefficient $\zeta_{\text {T,clean }}$ on a surfactant-free interface Eq. (17), the magnetic moment is determined by

$$
m=\frac{16 a\langle|U|\rangle}{3 \nabla B} .
$$

Figure 7(c) compares the magnetic moments for various microbuttons determined from measured resistances $\zeta_{R}$ and $\zeta_{T}$ in oscillation and translation modes at the air-water surface. The values from the two different calibrations methods agree very well, confirming that the two methods are consistent.

\section{Background magnetic field and apparent elasticity}

Any systematic background magnetic field $\left(B_{\text {sys }}\right)$ within the sample holder (e.g., due to the geomagnetic background, or to surrounding equipment with an unintended magnetization, or remnant magnetization of the iron core inserts) will act to align the ferromagnetic microbutton. The tendency of a probe to rotate, even in the absence of an applied field, would resemble the action of a surface elastic modulus, as calculated below. One must thus be careful to minimize the impact of systematic, background magnetic fields. To do so, we generally identify the direction of the systematic magnetic field, then rotate the sample holder such that axis 2 (the aligning field) is nearly parallel to the background field, and the axis of the driving electromagnets is perpendicular. In small-amplitude oscillatory modes, such an orientation ensures that $\left|\theta_{B}-\theta_{m}\right| \approx \pi / 2$, as in Fig. 8(a).

In an oscillatory rotational measurement, where $B_{s y s}$ is aligned perpendicular to the driving field $B_{0} \hat{\mathbf{e}}_{1}$, the systematic field exerts a restoring torque on the microbutton, in phase with the strain amplitude $\Delta \theta(t)$

$$
L_{\text {sys }}=m B_{\text {sys }} \sin (\Delta \theta(t)) \approx-m B_{\text {sys }} \Delta \theta(t) .
$$

This torque contributes what looks like an elastic response, even though it is completely unrelated to the sample rheology. The torque balance [Eq. (7)] reduces to

$$
i \omega \zeta_{R}^{*} \Delta \theta_{0}^{*} e^{i \omega t}=m B_{0}-m B_{s y s} \Delta \theta_{0}^{*}
$$

which can be expressed in the form

$$
\left(i \omega \zeta_{R}^{*}+m B_{s y s}\right) \Delta \theta_{0}^{*}=m B_{0} .
$$

Comparison with Eq. (7) reveals the systematic field giving an apparent rotational elastic constant

$$
k_{R}=m B_{\text {sys }},
$$

even though a clean Newtonian interface (e.g., water-air) exhibits no surface shear elasticity. An apparent elastic resistance $\zeta_{R}^{\prime \prime}$ thus appears in the rotational drag coefficient

$$
\zeta_{R}^{\prime \prime}(\omega)=-\frac{m B_{s y s}}{\omega} .
$$

Figure 8 reveals the effect of the systematic B-field on oscillatory measurements at a clean air-water surface. We

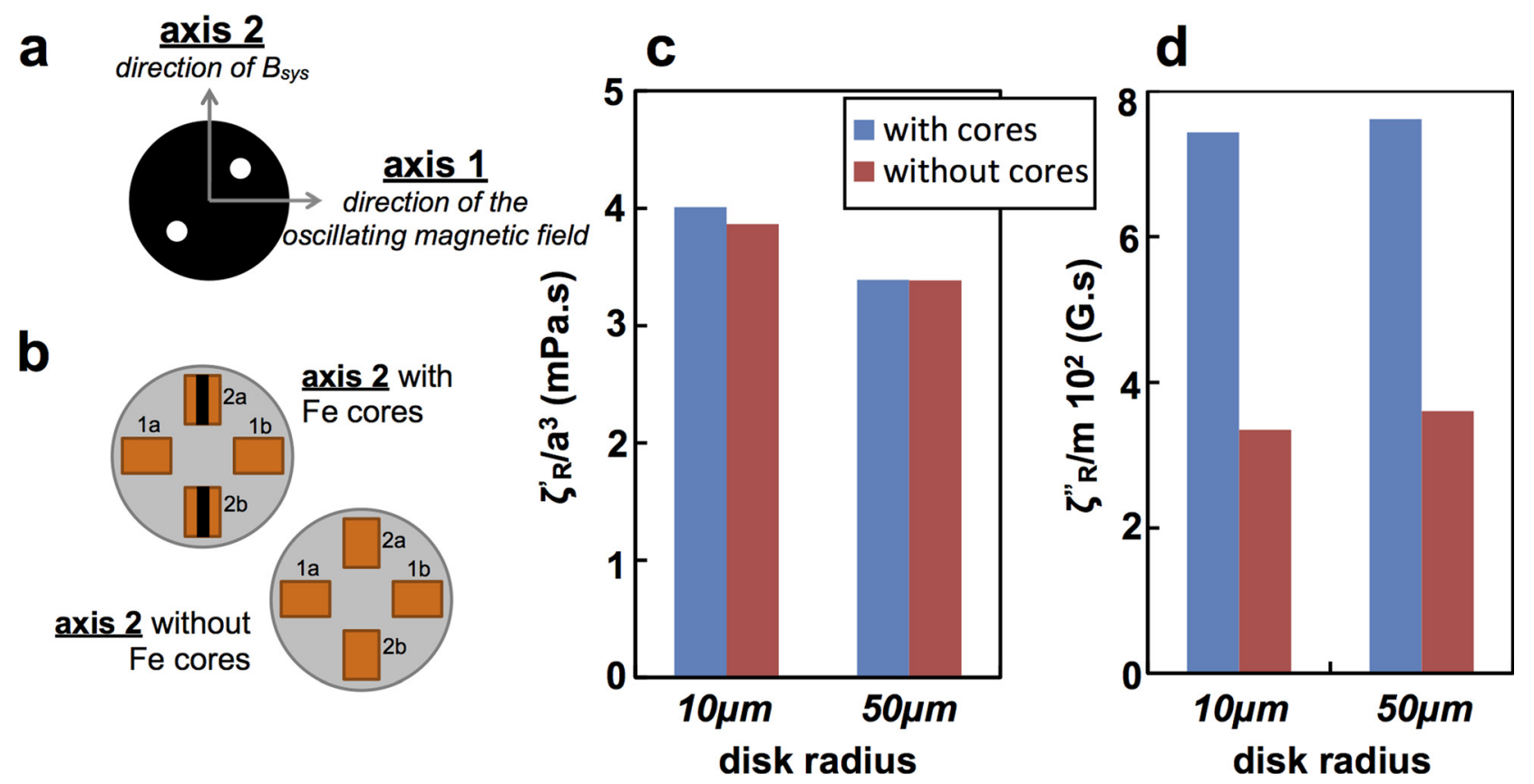

FIG. 8. (a) An oscillating magnetic field is imposed along the axis 1 . The sample holder is rotated to align axis 2 with the background magnetic field $B_{\text {sys. }}$ (b) Configurations with and without Fe cores inserted into axis 2 electromagnets are employed to determine the impact of $B_{\text {sys }}$ (c) and (d) Oscillatory measurements at $1 \mathrm{~Hz}$ using microbuttons of $10 \mu \mathrm{m} / 150 \mathrm{~nm} \mathrm{Ni}$ and $50 \mu \mathrm{m} / 150 \mathrm{~nm}$ Ni radii on a clean air-water interface, using electromagnets with and without Fe cores. (c) Normalized viscous $\operatorname{drag} \zeta_{R}^{\prime} / a^{3}$ and (d) normalized apparent elastic resistance $\zeta_{R}^{\prime \prime} / m$, which would be zero on a clean air-water interface in the absence of $B_{\text {sys }}$. The viscous drag $\zeta_{R}^{\prime}$ depends only on the disk radius $a$ and is independent of $B_{\text {sys. }}$. The apparent elastic resistance $\zeta_{R}^{\prime \prime}$ depends on the magnetic moment $m$ of the disk and on $B_{\text {sys }}$, which is larger when iron cores are inserted into electromagnet coils, due to residual magnetization. 
measure $\zeta_{R}^{\prime}$ and $\zeta_{R}^{\prime \prime}$ for two different sized microbuttons under two different configurations of the axis 2 electromagnets [Fig. 8(b)], with and without iron core inserts, whose remnant magnetization increases $B_{s y s}$. For both microbutton sizes, the viscous resistance $\zeta_{R}^{\prime}$ [Fig. 8(c)] is independent of $B_{\text {sys }}$ and consistent with $\zeta_{R}^{\prime}=16 \eta a^{3} / 3$, as expected for a surfactant-free air-water surface. By contrast, the rotational elastic resistance $\zeta_{R}^{\prime \prime}$ is nonzero, despite the known lack of surface elasticity for a clean Newtonian liquid. The measured $\zeta_{R}^{\prime \prime}$ is larger when iron core inserts are present [Fig. $8(\mathrm{~d})$ ], indicating $B_{s y s}$ is correspondingly larger. We determine $B_{s y s}$ using Eq. (30) to be approximately $0.3-0.4 \mathrm{G}$ without iron cores-i.e., comparable in magnitude to the geomagnetic field-and twice this value when cores are included.

\section{E. Measurement uncertainties}

The main source of uncertainty in microbutton rheometry arises due to the $15 \%$ and $20 \%$ variance in the measured magnetic moments of $10 \mu \mathrm{m}$ - and $50 \mu \mathrm{m}$-radius probes, respectively (Sec. IIIC). As the torque is directly proportional to the magnetic moment, this dispersion leads to 15\%-20\% errors in measured moduli. Ideally, one would measure the magnetic moment of a specific microbutton before using it for a rheology experiment; however, this is often impractical and at times impossible. Repeated measurements using different microbuttons can reduce the systematic uncertainties due to the uncertainty in the magnetic moment of each button.

A second source of uncertainty is introduced from finite precision in the spatial and temporal resolution with which $\Delta \theta_{0}^{*}$ and the applied voltage can be measured, which subsequently impacts the measured rotational resistance $\zeta_{R}^{*}$. Relative scatter in measured $\zeta_{R}^{*}$ for a given interface with a given microbutton is found to be of the order of $10 \%$ of $\zeta_{R}^{*}$ for any type of surface (e.g., for clean interface as well as for noninviscid monolayer). This error can be reduced by averaging measurements over larger numbers of oscillations, or by increasing the accuracy of strain tracking algorithms.

Uncertainties in measuring the phase angle $\delta$ become also particularly significant when the material response is either predominantly elastic $(\delta \approx 0)$ or viscous $(\delta \approx \pi / 2)$. In either case, the error on the subdominant modulus, $\Delta \zeta_{\text {sub }}$, appears as some fraction of the dominant modulus, $\Delta \zeta_{\text {sub }} / \zeta_{\text {dom }}=\Delta \delta$ where $\Delta \delta$ is the error on the phase lag. Using simulations of signals presenting noise comparable to experimental data, we estimated that $\Delta \delta \sim 0.01$ and is independent of the frequency. We therefore conclude that subdominant moduli more than 100 times weaker than the dominant modulus can not be reliably measured.

As with all microrheology, the accurate measurement of "macroscopically homogenized" rheological properties requires that the length scale over which the material is deformed be much larger than the material's inhomogeneities and/or microstructural elements. In some cases, the material microstructure can be directly visualized (e.g., grain boundaries in polycrystalline, liquid-condensed phospholipid monolayers, as studied by Choi and Squires [53], Choi et al. [54], and Kim et al. [55,56]), which either confirms or calls into question this separation of length scales. When material microstructures are not significantly smaller than the probe radius, making multiple measurements with multiple probe sizes can provide support for the validity of a given measurement (e.g., as in Kim et al. [56]).

Material heterogeneities and slip introduce another concern that can be easily alleviated if the material itself can be visualized. Zell et al. [59] used "gear"-shaped microbuttons to alleviate slip, and added tracer particles to map the strain field in the material. Using these strategies, we unambiguously demonstrated that the surface shear viscosity of sodium dodecyl sulfate (SDS) was below $10^{-8} \mathrm{~N} \mathrm{~s} / \mathrm{m}$, in conflict with decades of previous measurements. Interfacial strain visualization also confirmed that the flow in water+SDS/air interfaces is subphase dominated $(B o \ll 1)$. Finally, probe translation is relatively insensitive to probe/ material slip, and therefore provides a complementary technique to check measured values of $\zeta_{R}$ that seem suspiciously low.

\section{F. Performance limits and sensitivity}

We now discuss practical limits on the range of material properties that can be reliably measured using the microbutton rheometer for both interfacial shear rheology and bulk rheology. These limits are summarized in Table I.

Lower limits on the surface shear viscosity and elasticity that can be reliably measured are dominated by the $10 \%$ uncertainty on measured rotational resistance $\zeta_{R}^{*}$. $G_{s}^{*}(\omega)$ can only be determined unambiguously if $\zeta_{R}^{*}$ is sufficiently interfacially dominated ( $B o$ is sufficiently large) that these $10 \%$ uncertainties are not dominant.

The viscous drag coefficient $\zeta_{R}$ is dominated by the interfacial shear viscosity for $B o \gg 1$, which occurs when $\eta_{s}$

TABLE I. Dynamic range for surface shear rheological measurements for different microbutton probes assuming $B_{s y s} \sim 1 \mathrm{G}, B_{\max } \sim 120 \mathrm{G}, \theta_{\min } \sim 0.01 \mathrm{rad}$, and $f_{\min } \sim 0.1 \mathrm{~Hz}$.

\begin{tabular}{|c|c|c|c|c|c|c|c|c|c|}
\hline \multirow[b]{2}{*}{$\mathrm{a}(\mu \mathrm{m})$} & \multirow{2}{*}{$\begin{array}{c}\mathrm{Ni} \\
\text { thickness }(\mathrm{nm})\end{array}$} & \multirow[b]{2}{*}{$\mathrm{m}\left(10^{17} \mathrm{~J} / \mathrm{G}\right)$} & \multicolumn{4}{|c|}{ Surface rheology } & \multicolumn{3}{|c|}{ Bulk rheology } \\
\hline & & & $\eta_{s, \min }(\mu \mathrm{N} \mathrm{s} / \mathrm{m})$ & $G_{s, \min }^{\prime}(\mu \mathrm{N} / \mathrm{m})$ & $\left|G_{s}^{*}\right|_{\max }(\mu \mathrm{N} / \mathrm{m})$ & $\eta_{s, \max }(\mu \mathrm{N} \mathrm{s} / \mathrm{m})$ & $G_{\min }^{\prime}(\mathrm{Pa})$ & $\left|G^{*}\right|_{\max }(\mathrm{Pa})$ & $\eta_{\max }(\mathrm{Pa} \mathrm{s})$ \\
\hline 10 & 50 & 7 & 0.004 & 0.06 & 670 & 1100 & 0.01 & 160 & 250 \\
\hline 10 & 150 & 52 & 0.004 & 0.4 & 5000 & 7900 & 0.1 & 1200 & 1900 \\
\hline 50 & 150 & 970 & 0.02 & 0.3 & 3700 & 5900 & 0.015 & 170 & 280 \\
\hline
\end{tabular}


significantly exceeds a characteristic value, defined when $B o=1$ to be

$$
\eta_{s, \min }=\frac{4 \eta a}{3 \pi} .
$$

For microbuttons with 10 and $50 \mu \mathrm{m}$ radii on a water subphase, $\eta_{s, \min }$ is calculated equal to $4 \times 10^{-9}$ and $2 \times 10^{-8} \mathrm{~N}$ $\mathrm{s} / \mathrm{m}$, respectively. Experimentally, we find that $\eta_{s}$ can be clearly distinguished above measurement noise when it is a few times higher than $\eta_{s, \text { min }}$ (see Sec. IV C), giving a sensitivity around $\sim 10^{-8} \mathrm{~N} \mathrm{~s} / \mathrm{m}$ for $10 \mu \mathrm{m}$ probes. By comparison, the ISR has a sensitivity of $\eta_{s, \min } \sim 10^{-6} \mathrm{~N} \mathrm{~s} / \mathrm{m}$, the DWR has $\eta_{s, \text { min }} \sim 5 \times 10^{-6} \mathrm{~N} \mathrm{~s} / \mathrm{m}$, the deep-channel surface viscometer has $\eta_{s, \text { min }} \sim 10^{-7} \mathrm{~N} \mathrm{~s} / \mathrm{m}$, and the knife-edge viscometer reports $\eta_{s, \text { min }} \sim 10^{-8} \mathrm{~N} \mathrm{~s} / \mathrm{m}$ [5]. Torqued magnetic nanorods [46,74] and particle tracking microrheology $[36,37]$ have nominally higher sensitivity $\left(\eta_{s, \min } 10^{-9} \mathrm{~N} \mathrm{~s} / \mathrm{m}\right)$, but drive mixed rheological flows at the interface (i.e., with extension, compression, and dilation). These mixed flows complicate the quantitative extraction of nontrivial surface rheological properties in the most optimistic scenarios (i.e., interfaces that behave as truly incompressible and insoluble, and with interfacial contributions that significantly exceed the subphase contribution). When surfactants adsorb or desorb, or exhibit an (unknown) dilatational viscosity $\eta_{D}$, or behave as effectively incompressible at low apparent $B o$, then unambiguous interpretation of $\eta_{s}$ from a single measurement is not generally possible. Discrepancies between macroscopic (ISR) measurements and particle tracking microrheology can be quite strong [42,75], and are still not understood.

Any background magnetic field $B_{\text {sys }}$, discussed in Sec. IIID imposes an additional lower limit on the measurable surface elasticity. Background magnetic fields give an apparent (but false) surface elasticity

$$
G_{s, \min }^{\prime}=\frac{m B_{s y s}}{4 \pi a^{2}} .
$$

Taking $B_{\text {sys }}=1 \mathrm{G}$ as characteristic of the geomagnetic field gives a practical lower limit that can be achieved without magnetic shielding, in which case $G_{s, \min }^{\prime}=0.4$ and $0.3 \mu \mathrm{N} / \mathrm{m}$ for 10 and $50 \mu \mathrm{m}$ radius probes, respectively. Lower surface elasticities could be measured by reducing the magnetic moment of the probe (e.g., by reducing the thickness of the nickel layer, as in Table I, where $G_{s, \min }^{\prime}=0.05 \mu \mathrm{N} / \mathrm{m}$ for $50 \mathrm{~nm}$ Ni layers on $10 \mu \mathrm{m}$ probes).

Upper limits on measurement come from the maximum torque (and thus magnetic field) that can be applied, and from the minimum rotational displacement that can be measured reliably. In this case, the maximum surface modulus is given by

$$
\left|G_{s}^{*}\right|_{\max }=\frac{m B_{\max }}{4 \pi a^{2} \Delta \theta_{\min }},
$$

where $\Delta \theta_{\text {min }}=0.01 \mathrm{rad}$ is the minimum angular displacement that can be detected by the image analysis (Sec. IIE), and where $B_{\max }=120 \mathrm{G}$ is the maximum magnetic field strength that can be applied with the current electromagnets (with iron cores inserted). With these values, the maximum surface moduli measurable for standard $10 \mu \mathrm{m}$ and $50 \mu \mathrm{m}$ probes are $5000 \mu \mathrm{N} / \mathrm{m}$ and $3700 \mu \mathrm{N} / \mathrm{m}$, respectively.

Since the surface viscosity of purely viscous interfaces is given by $G_{s} / \omega$, the highest measurable surface viscosity is thus measured at the lowest practical frequency $f_{\text {min }}$. Convective and other experimental drift generally limits frequencies to $f_{\min }=0.1 \mathrm{~Hz}$, giving a maximum measurable surface viscosity

$$
\eta_{s, \text { max }}=\frac{m B_{\text {max }}}{8 \pi^{2} a^{2} f_{\text {min }} \Delta \theta_{\text {min }}}
$$

of order $10 \mathrm{mNs} / \mathrm{m}$.

There is no intrinsic lower limit to the bulk viscosity, other than that the probe must be suspended, and surface contamination and convective drift must be avoided. By working carefully, microbutton probes of any size can measure the viscosity of water ( $1 \mathrm{mPa} \mathrm{s})$. As discussed for minimum surface elasticity, the minimum measurable elastic modulus of the subphase is set by the "fake" elasticity that arises due to the background magnetic field $B_{\text {sys }}$. Using Eq. (14), for the rotational resistance of a thin disk at an interface, gives a minimum measurable $G_{\text {min }}^{\prime}$

$$
G_{\text {min }}^{\prime}=\frac{3 m B_{s y s}}{16 a^{3}},
$$

which ranges from 0.01 to $0.1 \mathrm{~Pa}$. Similarly, the maximum measurable modulus and viscosity are given by

$$
\begin{gathered}
\left|G^{*}\right|_{\max }=\frac{3 m B_{\max }}{16 a^{3} \Delta \theta_{\min }}, \\
\eta_{\max }=\frac{3 m B_{\max }}{32 \pi a^{3} f_{\min } \Delta \theta_{\min }},
\end{gathered}
$$

and range from $10^{2}$ to $10^{3} \mathrm{~Pa}$ and 100 to $1000 \mathrm{~Pa} \mathrm{~s}$, respectively, as summarized in Table I.

\section{SURFACE MICRORHEOMETRY}

Having described the operation, calibration, and validation of the technique, we now focus on its capabilities with demonstratory measurements on various material systems, each of which highlights a distinct qualitative capability. Previously, we have used microbuttons to measure the linear and nonlinear surface rheological properties of insoluble phospholipid monolayers [53-56], small-molecule soluble surfactants [59], the evolving rheology of drying suspensions [58], and the nonlinear rheology of yielding colloidal monolayers [57]. We demonstrate that microbuttons are capable of measuring broad types of surface rheology, including linear surface viscosity of Newtonian-like insoluble monolayers, nonlinear surface rheology of surface-yielding materials and aging of surfaces. Furthermore, the relatively quick measurements enable transient surface (and bulk) rheology to be 

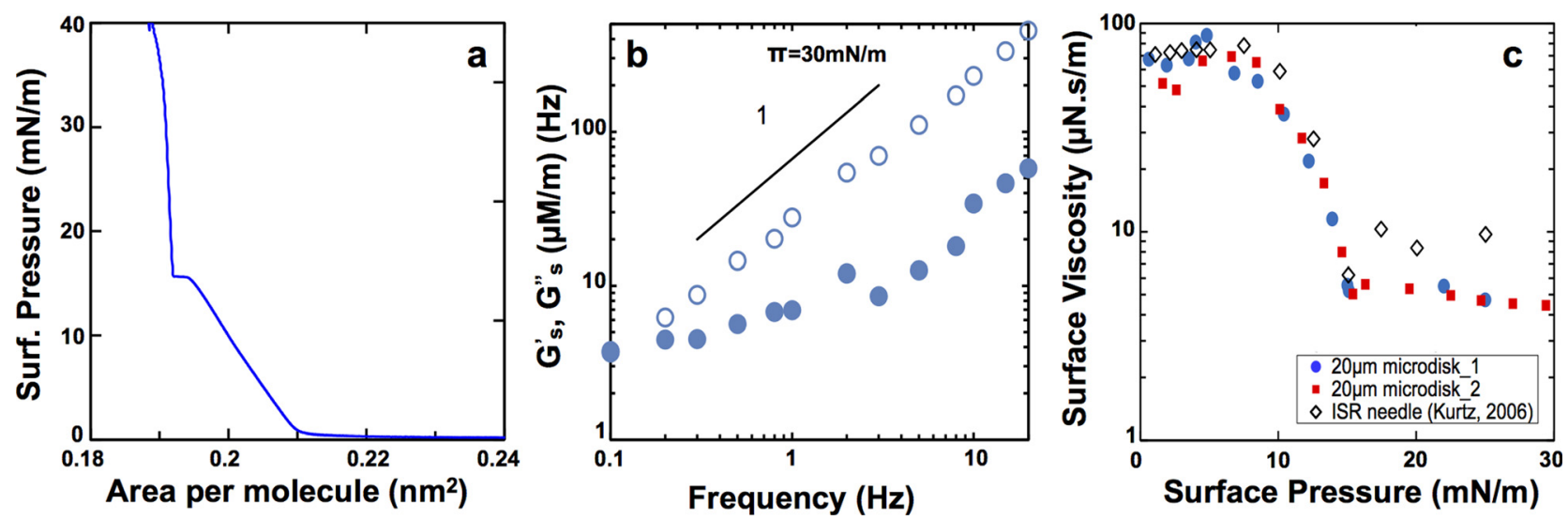

FIG. 9. (a) Surface pressure-area isotherm of an insoluble monolayer of 1-eicosanol. (b) Typical surface rheology $(\bigcirc) G_{s}^{\prime}$ and $(\bigcirc) G_{s}^{\prime \prime}$ vs. frequency for a 1eicosanol monolayer at surface pressure $\Pi=30.8 \mathrm{mN} / \mathrm{m}$. The 1-eicosanol monolayer behaves as a Newtonian interface with $G_{s}^{\prime \prime} \gg G_{s}^{\prime}$ and $G_{s}^{\prime \prime} \propto f$, and a surface viscosity $10^{3}$ times above the sensitivity limit of the microbutton rheometer. (c) Comparison between surface shear viscosity of 1-eicosanol monolayers measured in two distinct $10 \mu \mathrm{m}$ microbutton experiments $(\boldsymbol{\bullet})$ and with the ISR $(\diamond)$ [76] shows excellent agreement. Data from [59].

measured as the material evolves, with time scales ranging from a few minutes to several hours.

\section{A. Insoluble eicosanol monolayers}

We start with monolayers of 1-eicosanol, spread on the air-water interface, since these allow a direct comparison between our measurements and published measurements taken with the established ISR [19,76]. We spread 1eicosanol (Sigma Aldrich) from a chloroform solution (EMD Chemicals) onto deionized water (Millipore, $18.2 \mathrm{M} \Omega \mathrm{cm}$ ) in a Langmuir trough. The surface pressure-area (П-A) compression isotherm [Fig. 9(a)] measured with a Wilhelmy plate $(R \& K)$ shows two distinct phases, known to be a tilted, $L_{2}^{\prime}$ phase at low surface pressures, and an untilted, $L_{S, I}$ phase above $15 \mathrm{mN} / \mathrm{m}[19,76]$. A $10 \mu \mathrm{m}$ microbutton is added to the interface at a low surface pressure, and the surface is compressed to the desired surface pressure.

Figure 9(b) shows a typical surface shear rheology measurement in the $L_{S, I}$ phase: $\zeta_{R}$ is $10^{3}$ times above the sensitivity limit (confirming the measurement reflects an interfacially dominated system $B o \gg 1$ ), and the response is essentially Newtonian, with $G_{s}^{\prime \prime} \gg G_{s}^{\prime}$ and $G_{s}^{\prime \prime} \propto f$. Measuring $\eta_{s}=G_{s}^{\prime \prime} / \omega$ at $f=1 \mathrm{~Hz}$ while sweeping surface pressure [Fig. 9(c)] reveals quantitative agreement with published measurements taken with the ISR [19,76].

\section{B. PS-PEO block copolymer monolayers: Yielding}

We next examine a monolayer of polystyrene-b-poly(ethylene oxide) (PS-PEO) block copolymers spread at the air-water surface, which exhibits a nonlinear surface viscoelasticity. Previous ISR measurements of similar block copolymer monolayers have shown significant surface viscoelasticity at surface pressures above $8 \mathrm{mN} / \mathrm{m}$. Below this surface pressure, however, the measurement appeared to be subphase-limited [77]. We studied the equilibrium and dynamic behavior of a polystyrene-b-poly(ethylene oxide) block copolymer [PS(28.8 kg/ mol)-b-PEO (13.3 kg/mol), polydispersity index (PDI) 1.13 ] monolayer, spread from a dilute chloroform solution at the airwater surface. Atomic Force Microscopy (AFM) tapping mode imaging of the monolayer after Langmuir-Schaefer deposition at 2.6 and $8.0 \mathrm{mN} / \mathrm{m}$ [Figs. 10(a) and 10 (b)] reveals a microstructure of round, nanometer-scale surface aggregates, similar to those previously observed [77]. These structures form due to aggregation of the hydrophobic polystyrene block, and consist of a PS-rich core stabilized by the PEO block underneath and in the corona. At low concentrations, the surface pressure increases gradually as the film is compressed, followed by a pseudo first-order transition at intermediate pressures between $\sim 8-10 \mathrm{mN} / \mathrm{m}$. After the pseudoplateau, the surface pressure increases sharply with concentration, indicating hard-core repulsion between the aggregates [Fig. 10(c)].
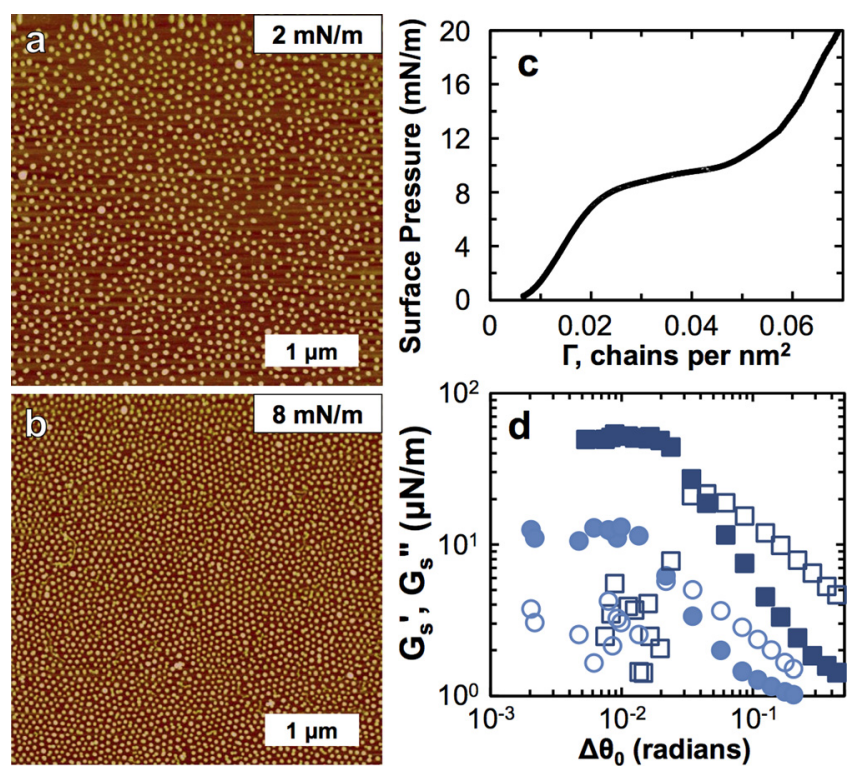

FIG. 10. AFM images of PS-PEO block copolymer monolayers transferred onto mica surfaces at surface pressures of (a) 2.6 and (b) $8.0 \mathrm{mN} / \mathrm{m}$. (c) Surface pressure-concentration isotherm of the monolayer shows transitions from a dilute phase at low $\Pi$, a pseudofirst order transition around $8 \mathrm{mN} / \mathrm{m}$, followed by a stiffening above $9 \mathrm{mN} / \mathrm{m}$ when aggregate cores start to overlap. (d) Dynamic strain sweeps at oscillation frequency $1 \mathrm{~Hz}$ of PS-PEO monolayers: Elastic (close symbols) and viscous (open symbols) surface moduli at surface pressures of $2.6(\bigcirc, \bullet)$ and $8.0(\square, \square) \mathrm{mN} / \mathrm{m}$. The measurements reveal a predominantly elastic response at low strains, followed by yielding (and viscous dominance) above a critical yield strain. Figures (a) and (b) reprinted with permission from Zell et al., Langmuir 30(1), 110-119 (2013). Copyright 2013, ACS Publication [89]. 
A linear-response oscillatory measurement reveals the monolayer to behave as predominantly elastic $\left(G_{s}^{\prime} \gg G_{s}^{\prime \prime}\right)$ at $1 \mathrm{~Hz}$ at both low $(2.6 \mathrm{mN} / \mathrm{m})$ and intermediate $(8.0 \mathrm{mN} / \mathrm{m})$ surface pressures shown in Figs. 10(a) and 10(b). Sweeping strain amplitudes at $1 \mathrm{~Hz}$, however, reveals qualitative differences in the nonlinear shear response. Strain-dependent moduli $G_{s}^{\prime}$ and $G_{s}^{\prime \prime}$ are obtained from a sinusoidal fit to the measured deformation response, and reveal rheological behavior consistent with surface yielding of a jammed 2D suspension of soft, repulsive particles [Fig. 10(d)]. At low strain amplitudes, the monolayer response is predominantly elastic. Beyond a critical yield strain of approximately $0.01-0.02 \mathrm{rad}$, the elastic component starts to decrease, and the viscous component increases, until the viscous response becomes dominant. At high amplitudes, both $G_{s}^{\prime}$ and $G_{s}^{\prime \prime}$ obey power law decreases, where $G_{s}^{\prime \prime}>G_{s}^{\prime}$. The surface moduli and surface yield strains grow with surface pressure, consistent with aggregates that become more densely packed, suggesting that the monolayer acts as a jammed 2D suspension of soft, repulsive particles [78].

\section{SDS: Transient rheology of aging interfaces}

Soluble surfactants are widely used in various industries to produce foams and stabilize emulsions. It has long been hypothesized that surface dynamic properties (as surface shear viscosities) correlate with foam and emulsion stability [1,2,5]. Our recent microbutton measurements [59], however, revealed the surface shear viscosity of a wide range of soluble surfactants to be immeasurably small (e.g., $\eta_{s} \leq$ $\eta_{s, \min } \sim 10^{-8} \mathrm{~N} \mathrm{~s} / \mathrm{m}$ ). This surprising surface shear inviscidity held even for SDS, for which many (contradictory) results had been reported previously [59,79-87]. During this study, however, we found the rheology of SDS interfaces to exhibit a strong and surprising aging over hour-long time scales. We hypothesize that multivalent cationic ions (e.g., introduced from sample holder walls) are responsible for this aging, and will explore in future work.

Here, we extract surface shear rheology from these SDS aging measurements, to demonstrate the sensitivity of the device. Figure 11(a) shows the (complex) rotational resistance $\zeta_{R}^{*}$ of a $10 \mu \mathrm{m}$ microdisk measured over more than $2 \mathrm{~h}$. At short times, the viscous $\zeta_{R}^{\prime}$ and elastic $\zeta_{R}^{\prime \prime}$ components remain
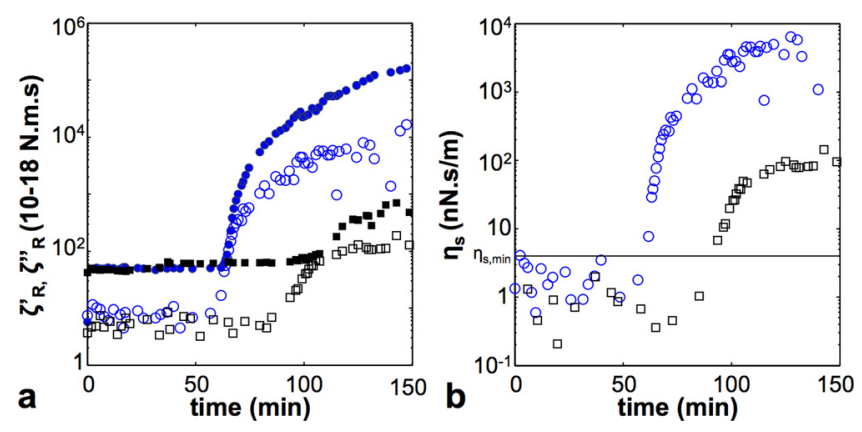

FIG. 11. (a) Rotational drag coefficient of a $10 \mu \mathrm{m}$ microdisk [viscous part $\zeta_{R}^{\prime}(\bigcirc, \square)$ and elastic part $\left.\zeta_{R}^{\prime \prime}(\boldsymbol{O}, \mathbf{\square})\right]$ and (b) surface viscosity $\eta_{s}$ as function of time for SDS solution with concentration of $3 \mathrm{mM}(\mathrm{O}, \mathbf{O})$ and $10 \mathrm{mM}$ $(\square, \mathbf{\square})$. Data from [59]. steady at $10^{-17}$ and $5 \times 10^{-17} \mathrm{~N} \mathrm{~s} / \mathrm{m}$, respectively. After 50-100 $\mathrm{min}$, however, $\left|\zeta_{R}^{*}\right|$ was measured to increase by several orders of magnitude.

Early values are consistent with subphase-dominated $\left(\eta_{s}^{a p p} \sim \eta_{s, \min }\right.$, or $\left.B o \sim 1\right)$ mechanics, so that the viscous and elastic rotational resistances are dominated by the subphase drag and the background magnetic field, respectively (see Secs. II D 1 and III D). Later values are consistent with interfacially dominated mechanics $(B o \gg 1)$, which can be interpreted using Eq. (12). The dependence of $\zeta_{R}^{*}$ upon $\eta_{s}$ and $\eta$ (i.e., for any $\mathrm{Bo}$ ) has been calculated for rotating disks by Hughes et al. [49], which we use to extract the apparent surface viscosity of the SDS interface as it evolves in time [Fig. 11(b)].

At short times, the apparent surface shear viscosity $\eta_{s}$ is very noisy, and of order $\eta_{s, \text { min }}=4 \mathrm{nN} \mathrm{s} / \mathrm{m}$ [Eq. (31)]. At these short times, one can conclude that any surface shear viscosity must be immeasurably small with our apparatus [59].

After 50-100 min, however, the apparent surface shear viscosity increases to a level that clearly emerges from measurement noise, and are clearly resolved once values of order $\sim 10^{-8} \mathrm{~N} \mathrm{~s} / \mathrm{m}$ are attained. This experiment confirms the $10^{-8} \mathrm{~N} \mathrm{~s} / \mathrm{m}$ sensitivity claimed in Sec. III E. Additionally, this experiment demonstrates that the microbutton is well suited for the measurement of transient surface rheology on systems that evolve on time scales of minutes to hours.

\section{Colloidal monolayers: Interfacial visualization}

Colloidal particles with intermediate wettability adsorb strongly to water/oil and water/air interfaces [88-90], and experience long-ranged repulsions due to unscreened electrostatic interactions through the oil phase [91-95]. Figure 12(a), for example, shows the repulsive, disordered solid monolayer that forms even in a low area-fraction $(\sim 0.5 \%)$ monolayer of $0.9 \mu \mathrm{m}$-radius colloids adsorbed to a waterdecane interface. Small-amplitude, oscillatory microbutton measurements of this monolayer reveal an immeasurably small surface shear rheology, with drag dominated by the water and decane subphases. When the microbutton is translated, however, the colloidal monolayer changes even the qualitative nature of the flow field, despite its lack of surface shear viscosity. As highlighted by Fischer [68] and [48] and demonstrated experimentally [62,96], even surface-shear inviscid surfactant monolayers act to maintain 2D incompressibility, which fundamentally changes the boundary conditions imposed on the subphase fluid flows, and therefore changes the translational resistance $\zeta_{T}$ from its value at a "clean" interface. Direct visualization of the 2D flow field around a probe translating within a colloidal monolayer confirms this 2D incompressibility.

The colloidal monolayer is prepared following standard techniques [90]. Polystyrene microbeads with $1.8 \mu \mathrm{m}$ diameter (Bangs laboratories, Inc.) are dispersed in a $50 \% \mathrm{w}-50 \% \mathrm{w}$ isopropyl alcohol-water solution. A few drops of the dispersion are deposited on a deionized water surface, and rapidly spread across the surface to leave a monolayer of adsorbed particles. A $50 \mu \mathrm{m}$ radius microbutton is then 

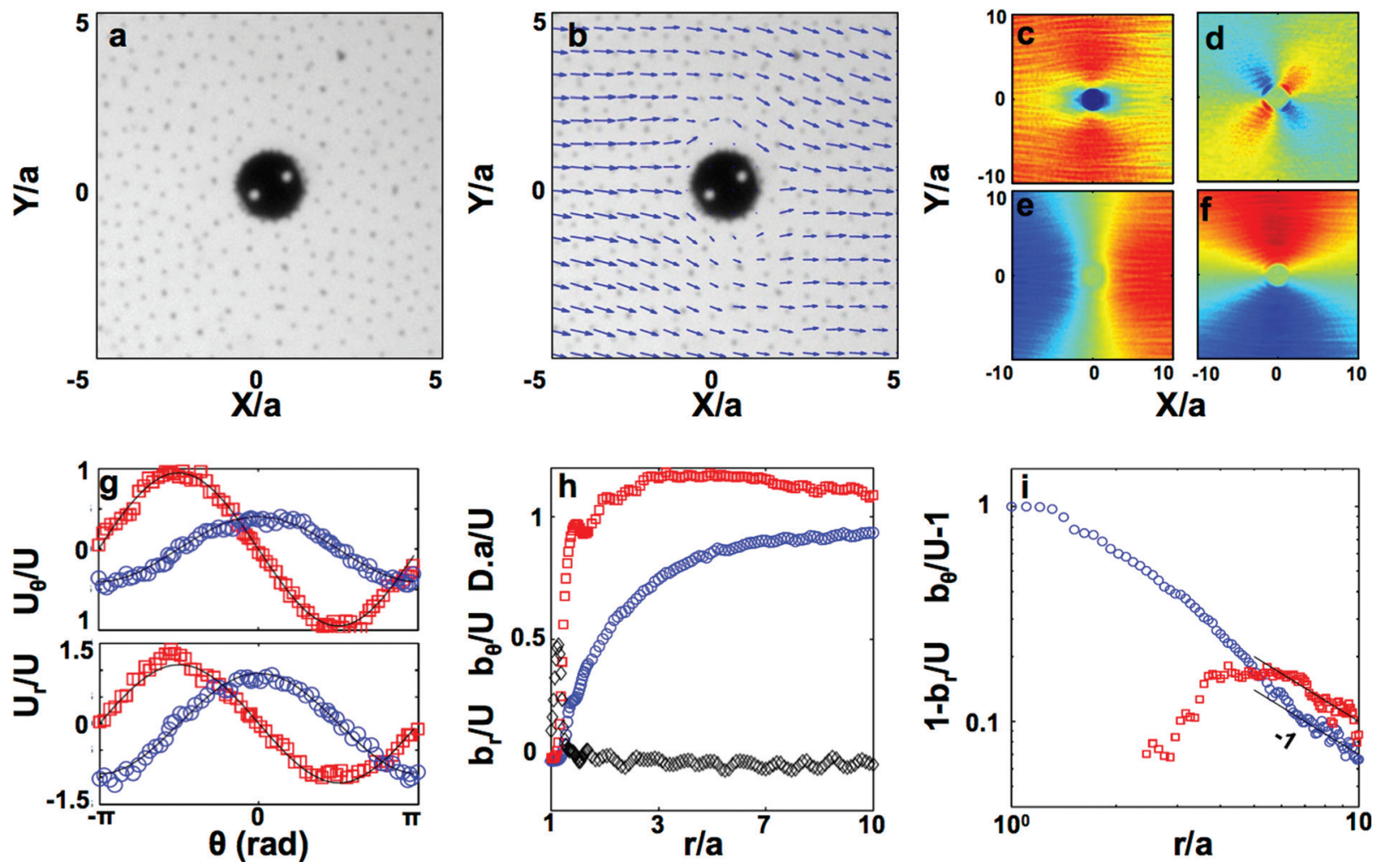

FIG. 12. (a) A 2D suspension of $1.8 \mu \mathrm{m}$ diameter polystyrene colloids adsorbed to a water-decane interface, within which a $50 \mu \mathrm{m}$ microdisk is forced to translate from right to left. (b) Measured surface velocity field during microbutton translation, viewed in the frame co-moving with the probe. (c)-(f) Velocity components as a function of position. (c) Velocity component in the x-direction; (d) y-direction; (e) radial ( $r$-)direction; and (f) azimuthal ( $\theta$-)direction. (g) Radial $(\bigcirc)$ and azimuthal $(\square)$ velocities vs azimuthal angle $\theta$ at two different distances from the disk center: 2 disk radii (top) and 10 disk radii (bottom). Black lines represent sinusoidal fits. (h) Normalized values of the prefactors $b_{r}(\bigcirc), b_{\theta}(\square)$ and surface divergence $D(\diamond)$ as defined by Eqs. (38)-(40), as functions of the normalized distance from the disk center. (i) $1-b_{r} / U(\bigcirc)$ and $b_{\theta} / U-1(\square)$ as a function of the normalized distance from the disk center. Lines: Power law fit with an exponent of -1 .

adsorbed to the interface, decane is gently added on top of the microbutton/colloid/water surface, and the system is allowed to equilibrate for several minutes before performing experiments. Figure 12(a) shows a monolayer with 980 particles $/ \mathrm{mm}^{2}$, corresponding to a $0.5 \%$ area fraction.

The microbutton is forced into translation with an imposed magnetic field gradient (Sec. III D), and dynamics within the colloidal monolayer are captured using videomicroscopy. We measure the surface velocity field $\vec{u}_{s}$ [Figs. 12(b)-12(d)] through image analysis in MATLAB, using a method that works for any system with an optically heterogeneous interface. We first detect the center of the microdisk using the function imfindcircles in MATLAB (Sec. IIE), then crop the image to a region of fixed size, centered on the probe, so that we measure a velocity field in a reference frame fixed on the probe. We then use the optical flow method [97] to determine surface velocity vectors at each point on the interface (averaged over 300 image pairs), measured in a reference frame co-moving with the probe. For simplicity of presentation, we rotate the image frame so that probe translation occurs along the $\mathrm{x}$-axis.

We convert these velocities to cylindrical coordinates to obtain radial and azimuthal velocities $u_{r}$ and $u_{\theta}$ [Figs. 12(e) and 12(f)], which symmetry [49] and measurements [Fig. $12(\mathrm{~g})]$ take the form

$$
u_{r}=b_{r}(r) \cos \theta,
$$

$$
u_{\theta}=-b_{\theta}(r) \sin \theta .
$$

Here, $b_{r}$ and $b_{\theta}$ are functions of the distance from disk center [Fig. 12(h)]. The surface velocities (and thus $b_{r}$ and $b_{\theta}$ ) should vanish at the microbutton radius of the disk, corresponding to the nonslip condition, and should approach constant values far from the probe, corresponding to the uniform flow $b_{r}(r \rightarrow \infty)=b_{\theta}(r \rightarrow \infty)=U$. Here, however, we notice that $b_{\theta}$ increases rapidly over the first $20 \mu \mathrm{m}$, corresponding to the distance between the edge of the probe and the first layer of particles, reaches a maximum at $r=250 \mu \mathrm{m}$, then decreases to approach the constant $U$.

The flow field on a clean interface far from the probe can be determined from the point force solution, whose form would be given by Eqs. (38) and (39) with $\left(U-b_{r}\right)$ $\sim\left(U-b_{\theta}\right) \sim 1 / r$ [98]. Such scaling is indeed observed experimentally, although one would expect both $b_{r}$ and $b_{\theta}$ to stay lower than $U$.

Following [48,68], we compute the surface divergence of the surface velocity field measured around the translating probe, which is given by

$$
\vec{\nabla} \cdot \vec{u}_{s}=\cos \theta \frac{1}{r}\left(\frac{d}{d r}\left(r b_{r}\right)-b_{\theta}\right) \equiv \cos \theta D(r),
$$

and plotted in Fig. 12(h). Within the first ring of particles (i.e., within $20 \mu \mathrm{m}$ of the probe), the surface divergence is 
nonzero, with a compression zone in front of the probe and a dilation zone behind it. Beyond the first ring of particles, however, the surface divergence $D(r)$ vanishes remarkably quickly, indicating neither surface compression nor dilatation. The surface divergence field on a clean (stress-free) fluid interface, by contrast, has large zones of compression and dilation around a translating probe.

In conclusion, the colloidal monolayer highlighted in Fig. 12 gives rise to an immeasurably small surface shear viscosity, but acts to maintain 2D incompressibility of the interface. With the particulate monolayer, compression/dilation occurs only immediately adjacent to the probe, in a region devoid of particles, which one can attribute to noncontinuum effects of the monolayer. This 2D incompressibility modifies the subphase velocity field when the surface is deformed by a nonpure shear flow, relative to what would be established if the interface were clean. Such effects can significantly complicate the interpretation of surface rheology measurements from any experiments establishing nonviscometric flows. We argued that such effects could contribute to the wide range of surface shear viscosities previously measured for SDS and other soluble surfactants [59], despite their surface shear viscosities being immeasurably low.

\section{BULK MATERIAL RHEOLOGY}

Although we originally developed the microbutton for interfacial shear rheometry, we have also used them to measure the rheology of bulk materials. As with more conventional (particlebased) microrheometry, the small size of microbuttons enable rheological measurements on small $(\sim \mu \mathrm{l})$ sample volumes. Moreover, active microbutton forcing enables its use in interrogating higher-modulus materials that would be impossible (or impractical) with passive (thermal) rheometry. The frequency range accessible to microbuttons is, of course, far smaller than for passive microrheology, and other limitations hold. We anticipate that such capabilities will be useful for measurements of small and precious samples, as well as evolving materials [58]. To demonstrate these capabilities, we present several proof-of-principle measurements of bulk material rheology: Simple Newtonian liquids, viscoelastic solutions, and measurements with microliter-scale sample volumes.

\section{A. Frequency-dependent viscoelasticity: Xanthan gum solutions}

We compare the frequency-dependent viscoelastic moduli of aqueous xanthan gum solutions, as measured using a macroscopic rheometer and microbutton. We prepared solutions by dissolving Xanthan gum powder (Vanzan, Vanderbilt Materials) in deionized water (Milli-pore, $18 \mathrm{M} \Omega \mathrm{cm}$ ) and mixing with a magnetic stir bar until fully dispersed. We pipetted approximately $2 \mathrm{ml}$ of solution into the aluminum sample holder [Fig. 2(b)], and placed a $50 \mu \mathrm{m}$ microbutton on the solution surface. For comparison, we performed dynamic frequency sweep measurements using the microbutton and macroscopic AR-G2 rheometer $(40 \mathrm{~mm}$ parallel plate geometry, $1 \mathrm{~mm}$ gap). Although xanthan gum solutions remain in the linear viscoelastic regime until more than
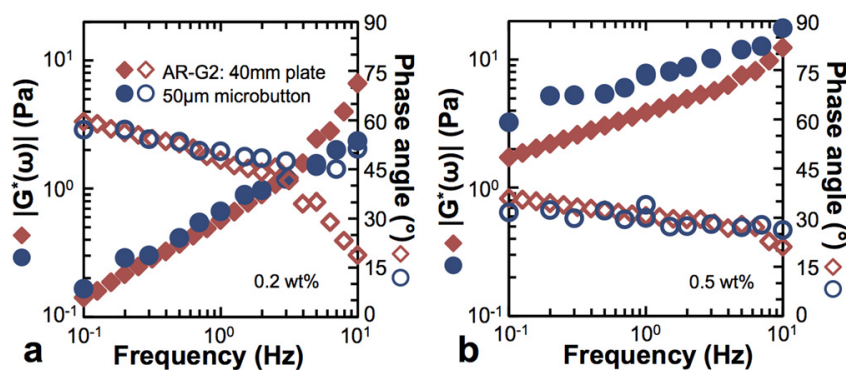

FIG. 13. The magnitude of measured shear moduli (closed) and phase angle (open) of (a) $0.2 \mathrm{wt} . \%$ and (b) $0.5 \mathrm{wt} . \%$ Xanthan gum solutions as measured using $50 \mu \mathrm{m}$ microbutton microrheometry $(\mathrm{O}, \bullet)$ and AR-G2 parallel plate geometry $(\diamond, \diamond)$. The measurements using the microbutton are in good agreement with the macroscopic measurements.

$100 \%$ strain amplitude [99], we applied relatively low strain amplitudes of $0.05 \mathrm{rad}$ (microbutton) and 1\% (AR-G2).

Both micro- and macromeasurements reveal viscousdominant moduli at frequencies below $3 \mathrm{~Hz}$ for $0.2 \%$ solutions of xanthan gum. Measurements of the bulk modulus $\left(\left|G^{*}(\omega)\right|\right)$ and the phase angle $\delta$ made by both techniques agree very well at these frequencies [Fig. 13(a)]. Above $3 \mathrm{~Hz}$, a small but growing deviation between the two methods appears.

More concentrated $(0.5$ wt. \%) xanthan gum solutions exhibit an elastic-dominant response over the entire frequency range probed. Broad agreement is evident between the two methods of rheology [Fig. 13(b)], with phase angle measurements in quantitative agreement, and modulus measurements that grow with frequency in the same fashion. Nevertheless, the microbutton measures a complex modulus approximately double that measured by the rheometer. This may reflect a low magnetic moment for that particular microbutton (see data spread in Fig. 7), surface-active materials to which the microbutton is more sensitive, local heterogeneities in the microrheological measurements, or changes to the material microstructure related to the presence of the interface.

More broadly, however, microbutton measurements are generally in excellent agreement with macroscopic rheometry for these Xanthan gum solutions, confirming their potential utility in bulk material rheometry.

\section{B. Rheology of microliter volume samples}

We finally note that the small size of microbuttons enables rheometry on the microliter volume scale, as would be required for screens of newly synthesized compounds and formulations (e.g., in the pharmaceutical industry). To demonstrate, we measured the viscosity of $2 \mu \mathrm{l}$ sample volumes of glucose solutions ranging from $0 \%$ to $50 \mathrm{wt}$. $\%$, and sucrose solutions from $0 \%$ to $70 \mathrm{wt}$. \%, covering a wide range of viscosities between 1 and $500 \mathrm{mPa}$.

While more elaborate sample holders could certainly be made, we performed proof-of-principle measurements using a hanging drop (inset of Fig. 14). We create a trap to hold the drop in place by cutting a 2-mm-diameter circular hole through $50-\mu \mathrm{m}$-thick scotch tape, which is then applied to a glass slide. Using a microsyringe, we deposit $2 \mu \mathrm{l}$ of solution on the glass slide in the middle of the hole. Using a plastic pipette tip, we deposit a microbutton onto the drop, taking care to ensure that both the tip and the button are dried to 


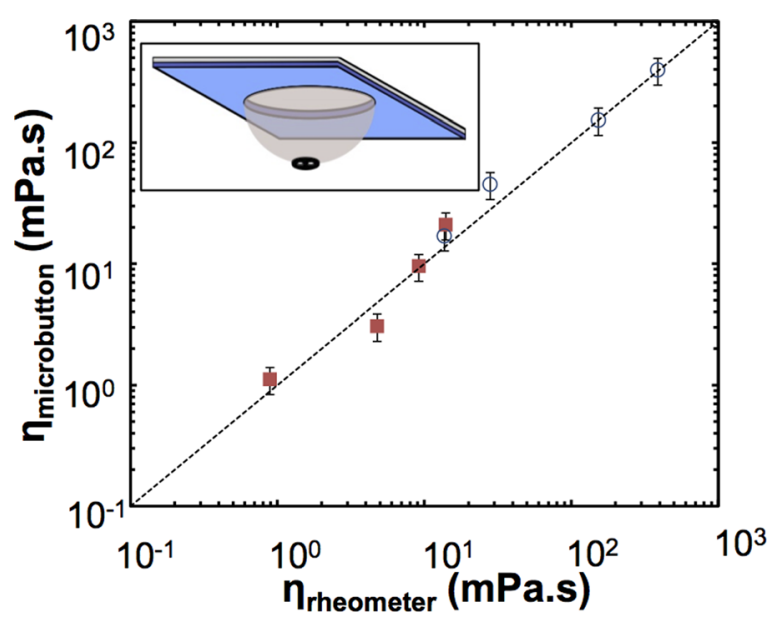

FIG. 14. Viscosity measured with microbuttons is plotted against that measured using a cone and plate rheometer, for (ם) $0 \%-50_{w t} \%$ glucose solutions and $(\bigcirc) 0 \%-70_{w t} \%$ sucrose solutions.

avoid contaminating the drop. We then flip the glass slide so that the drop hangs, whereupon gravity pulls the microbutton to settle at the bottom of the drop. We then measure the viscoelastic moduli as described above. Excellent agreement is found between the viscosities measured using the microbutton and cone and plate rheometry (Fig. 14), confirming that the microbutton technique is suitable for measurements on microliter volumes of precious sample.

\section{CONCLUSION}

We developed a novel microrheometer to actively measure rheological properties of different materials. This microrheometer has been used in a series of recent articles to study interfacial rheology [52-56,58,59]. Here, we extensively described the experimental apparatus and discussed data analysis so that interested readers can duplicate and use the microrheometer for their own purposes.

The microbutton rheometer has numerous capabilities, as demonstrated through a series of experiments presented here. Measurements of interfacial and bulk rheology obtained using the microrheometer are in quantitative agreement with those obtained using other interfacial and bulk rheometers. Oscillatory linear rheology and nonlinear shear rheology can also be measured using the microrheometer. Additionally, the method allows a direct visualization of the interface in order to correlate rheological measurements with morphological processes.

The combined versatility and sensitivity of the microrheometer makes it an important addition to the suite of tools in current use for bulk and interfacial rheometry. The method is particularly well suited to study materials with low surface viscosities, complex surface rheology or transient rheology, or to study materials that can only be obtained in small sample volumes.

\section{ACKNOWLEDGMENTS}

The authors gratefully acknowledge the support from the National Science Foundation under Grant No. CBET-932259
(Z.Z.), the NSF MRSEC program under Award No. DMR0520415 (S.Q.C.), the National Institutes of Health under Grant No. HL-51177 (K.K.), the Camille and Henry Dreyfus Teacher-Scholar program (J.W.), and the Dow Chemical Company through the Dow Materials Institute at UCSB (V.M. and Z.Z.). Work was performed in UCSB's Materials Research Laboratory Central Facilities, which are supported by the NSF Materials Research Science and Engineering Centers Program under Grant No. DMR 1121053, a member of the NSF-funded Materials Research Facilities Network, and in the UCSB Nanofabrication Facility, part of the NSFfunded National Nanotechnology Infrastructure Network.

\section{References}

[1] Tadros, T. F., Surfactants in Nano-Emulsions. Applied Surfactants: Principles and Applications (John Wiley \& Sons, Weinheim, Germany, 2005), pp. 285-308.

[2] Schramm, L. L., Emulsions, Foams, and Suspensions: Fundamentals and Applications (John Wiley \& Sons, Weinheim, Germany, 2006).

[3] Kaganer, V. M., H. Mohwald, and P. Dutta, "Structure and phase transitions in Langmuir monolayers," Rev. Mod. Phys. 71(3), 779-819 (1999).

[4] Sagis, L. M. C., "Dynamic properties of interfaces in soft matter: Experiments and theory," Rev. Mod. Phys. 83(4), 1367-1403 (2011).

[5] Edwards, D. A., H. Brenner, and D. T. Wasan, Interfacial Transport Processes and Rheology (Butterworth-Heinemein, Stonheam, MA, 1991).

[6] Fuller, G. G., and J. Vermant, "Complex fluid-fluid interfaces: Rheology and structure," Ann. Rev. Chem. Biomol. Eng. 3(1), 519-543 (2012).

[7] Langevin, D., "Influence of interfacial rheology on foam and emulsion properties," Adv. Colloid Interface Sci. 88(1), 209-222 (2000).

[8] Scriven, L. E., "Dynamics of a fluid interface equation of motion for Newtonian surface fluids," Chem. Eng. Sci. 12, 98-108 (1960).

[9] Harkins, W. D., and R. J. Myers, "Viscosity of monomolecular films," Nature 140, 465 (1937).

[10] Myers, R. J., and W. D. Harkins, "The viscosity (or fluidity) of liquid or plastic monomolecular films," J. Chem. Phys. 5(7), 601-603 (1937).

[11] Harkins, W. D., and J. G. Kirkwood, "The viscosity of monolayers: Theory of the surface slit viscometer," J. Chem. Phys. 6(1), 53 (1938).

[12] Sacchetti, M., H. Yu, and G. Zografi, "A canal surface viscometer for the in plane steady shear viscosity of monolayers at the air/water interface,” Rev. Sci. Instrum. 64(7), 1941-1946 (1993).

[13] Burton, R. A., and R. J. Mannheimer, "Analysis and apparatus for surface rheological measurements in ordered fluids and liquid crystals," Adv. Chem. Ser. 63, 315-328 (1967).

[14] Mannheimer, R. J., "Surface rheological properties of foam stabilizers in nonaqueous liquids," AIChE J. 15(1), 88-93 (1969).

[15] Mannheimer,R. J., and R. S. Schechter, "An improved apparatus and analysis for surface rheological measurements," J. Colloid Interface Sci. 32(2), 195-211 (1970).

[16] Pintar, A. J., A. B. Israel, and D. T. Wasan, "Interfacial shear viscosity phenomena in solutions of macromolecules," J. Colloid Interface Sci. 37(1), 52-67 (1971).

[17] Goodrich, F. C., L. H. Allen, and A. Poskanzer, "A new surface viscometer of high sensitivity. I. Theory,” J. Colloid Interface Sci. 52(2), 201-212 (1975).

[18] Poskanzer, A., and F. C. Goodrich, "A new surface viscometer of high sensitivity. II. Experiments with stearic acid monolayers," J. Colloid Interface Sci. 52(2), 213-221 (1975). 
[19] Brooks, C. F., G. G. Fuller, C. W. Frank, and C. R. Robertson, "An interfacial stress rheometer to study rheological transitions in monolayers at the air-water interface," Langmuir 15(7), 2450-2459 (1999).

[20] Ding, J., H. E. Warriner, J. A. Zasadzinski, and D. K. Schwartz, "Magnetic needle viscometer for Langmuir monolayers," Langmuir 18(7), 2800-2806 (2002).

[21] Reynaert, S., C. F. Brooks, P. Moldenaers, J. Vermant, and G. G. Fuller, "Analysis of the magnetic rod interfacial stress rheometer," J. Rheol. 52(1), 261-285 (2008).

[22] Goodrich, F. C., and A. K. Chatterjee, "The theory of absolute surface shear viscosity II. The rotating disk problem,” J. Colloid Interface Sci. 34(1), 36-42 (1970).

[23] Boyd, J., and P. Sherman, "Two-dimensional rheological studies on surfactant films at interfaces,” J. Colloid Interface Sci. 34(1), 76-80 (1970).

[24] Gaub,H. E., and H. M. McConnell, "Reversible formation of plastic two-dimensional lipid crystals," J. Phys. Chem. 90(26), 6830-6832 (1986).

[25] Soo-Gun, O., and J. C. Slattery, "Disk and biconical interfacial viscometers," J. Colloid Interface Sci. 67(3), 516-525 (1978).

[26] Dickinson, E., B. S. Murray, and G. Stainsby, "Time dependent surface viscosity of adsorbed films of casein + gelatin at the oil water interface," J. Colloid Interface Sci. 106(1), 259-262 (1985).

[27] Erni, P., P. Fischer, E. J. Windhab, V. Kusnezov, H. Stettin, and J. Lauger, "Stress-and strain-controlled measurements of interfacial shear viscosity and viscoelasticity at liquid/liquid and gas/liquid interfaces," Rev. Sci. Instrum. 74(11), 4916-4924 (2003).

[28] Luap, C., and W. A. Goedel, "Linear viscoelastic behavior of endtethered polymer monolayers at the air/water interface," Macromolecules 34(5), 1343-1351 (2001).

[29] Franck, A., "Grenzflchencharakterisierung mit dem du noy ring," Nachrichten Chem. 55(2), 163-164 (2007).

[30] Vandebril, S., A. Franck, G. G. Fuller, P. Moldenaers, and J. Vermant, "A double wall-ring geometry for interfacial shear rheometry," Rheol. Acta 49(2), 131-144 (2010).

[31] Mason, T. G., and D. A. Weitz, "Optical measurements of frequencydependent linear viscoelastic moduli of complex fluids," Phys. Rev. Lett. 74(7), 1250-1253 (1995).

[32] Mason, T. G., K. Ganesan, J. H. van Zanten, D. Wirtz, and S. C. Kuo, "Particle tracking microrheology of complex fluids," Phys. Rev. Lett. 79(17), 3282-3285 (1997).

[33] Gittes, F., B. Schnurr, P. D. Olmsted, F. C. MacKintosh, and C. F. Schmidt, "Microscopic viscoelasticity: Shear moduli of soft materials determined from thermal fluctuations," Phys. Rev. Lett. 79(17), 3286-3289 (1997).

[34] Squires, T. M., and T. G. Mason, "Tensorial generalized Stokes Einstein relation for anisotropic probe microrheology," Annu. Rev. Fluid Mech. 42(1), 413-438 (2010).

[35] Sickert, M., and F. Rondelez, "Shear viscosity of Langmuir monolayers in the low-density limit," Phys. Rev. Lett. 90(12), 126104 (2003).

[36] Prasad, V., S. A. Koehler, and E. R. Weeks, "Two-particle microrheology of quasi-2D viscous systems," Phys. Rev. Lett. 97(17), 176001 (2006).

[37] Sickert, M., F. Rondelez, and H. A. Stone, "Single-particle Brownian dynamics for characterizing the rheology of fluid Langmuir monolayers," Europhys. Lett. 79(6), 66005 (2007).

[38] Prasad, V., and E. R. Weeks, "Flow fields in soap films: Relating viscosity and film thickness," Phys. Rev. E 80(2), 026309 (2009).

[39] Prasad, V., and E. R. Weeks, "Two-dimensional to three-dimensional transition in soap films demonstrated by microrheology," Phys. Rev. Lett. 102(17), 178302 (2009).
[40] Ortega, F., H. Ritacco, and R. G. Rubio, "Interfacial microrheology: particle tracking and related techniques," Curr. Opin. Colloid Interface Sci. 15(4), 237-245 (2010).

[41] Kandar, A. K., R. Bhattacharya, and J. K. Basu, "Interfacial microrheology as a tool to study viscoelastic transitions in nanoconfined soft matter," Phys. Rev. E 81(4), 041504 (2010).

[42] Maestro, A., L. J. Bonales, H. Ritacco, T. M. Fischer, R. G. Rubio, and F. Ortega, "Surface rheology: Macro-and microrheology of poly (tertbutyl acrylate) monolayers," Soft Matter 7(17), 7761-7771 (2011).

[43] Lee, M. H., S. P. Cardinali, D. H. Reich, K. J. Stebe, and R. L. Leheny, "Brownian dynamics of colloidal probes during protein-layer formation at an oil water interface," Soft Matter 7(17), 7635-7642 (2011).

[44] Lee, M. H., D. H. Reich, K. J. Stebe, and R. L. Leheny, "Combined passive and active microrheology study of protein-layer formation at an air water interface," Langmuir 26(4), 2650-2658 (2009).

[45] Breedveld, V., and D. J. Pine, "Microrheology as a tool for highthroughput screening," J. Mater. Sci. 38(22), 4461-4470 (2003).

[46] Dhar, P., Y. Cao, T. M. Fischer, and J. A. Zasadzinski, "Active interfacial shear microrheology of aging protein films," Phys. Rev. Lett. 104(1), 016001 (2010).

[47] Erni, P., M. Varagnat, C. Clasen, J. Crest, and G. H. McKinley, "Microrheometry of sub-nanolitre biopolymer samples: NonNewtonian flow phenomena of carnivorous plant mucilage," Soft Matter 7(22), 10889-10898 (2011).

[48] Fischer, T. M., P. Dhar, and P. Heinig, "The viscous drag of spheres and filaments moving in membranes or monolayers," J. Fluid Mech. 558, 451-475 (2006).

[49] Hughes, B. D., B. A. Pailthorpe, and L. R. White, "The translational and rotational drag on a cylinder moving in a membrane," J. Fluid Mech. 110, 349-372 (1981).

[50] Levine, A. J., T. B. Liverpool, and F. C. MacKintosh, "Dynamics of rigid and flexible extended bodies in viscous films and membranes," Phys. Rev. Lett. 93(3), 038102 (2004).

[51] de Gennes, P. G., "Wetting: Statics and dynamics," Rev. Mod. Phys. 57(3), 827-863 (1985).

[52] Choi, S. Q., S. G. Jang, A. J. Pascall, M. D. Dimitriou, T. Kang, C. J. Hawker, and T. M. Squires, "Synthesis of multifunctional micrometer sized particles with magnetic, amphiphilic, and anisotropic properties," Adv. Mater. 23(20), 2348-2352 (2011).

[53] Choi, S. Q., and T. M. Squires, "Dynamics within surfactant monolayers," Phys. Fluids 22(9), 091113 (2010).

[54] Choi, S. Q., S. Steltenkamp, J. A. Zasadzinski, and T. M. Squires, "Active microrheology and simultaneous visualization of sheared phospholipid monolayers," Nat. Commun. 2, 312 (2011).

[55] Kim, K., S. Q. Choi, J. A. Zasadzinski, and T. M. Squires, "Interfacial microrheology of DPPC monolayers at the air water interface," Soft Matter 7(17), 7782-7789 (2011).

[56] Kim, K., S. Q. Choi, Z. A. Zell, T. M. Squires, and J. A. Zasadzinski, "Effect of cholesterol nanodomains on monolayer morphology and dynamics," Proc. Natl. Acad. Sci. U.S.A. 110, E3054-E3060 (2013).

[57] Buttinoni, I., Z. A. Zell, T. M. Squires, and L. Isa, "Colloidal binary mixtures at fluid-fluid interfaces under steady shear: Structural, dynamical and mechanical response," Soft Matter 11(42), 8313-8321 (2015).

[58] Komoda, Y., L. G. Leal, and T. M. Squires, "Local, real-time measurement of drying films of aqueous polymer solutions using active microrheology," Langmuir 30(18), 5230-5237 (2014).

[59] Zell, Z. A., A. Nowbahar, V. Mansard, L. G. Leal, S. S. Deshmukh, J. M. Mecca, C. J. Tucker, and T. M. Squires, "Surface shear inviscidity of soluble surfactants," Proc. Natl. Acad. Sci. U.S.A. 111(10), 3677-3682 (2014). 
[60] Badaire, S., C. Cottin-Bizonne, J. W. Woody, A. Yang, and A. D. Stroock, "Shape selectivity in the assembly of lithographically designed colloidal particles," J. Am. Chem. Soc. 129(1), 40-41 (2007).

[61] Hernandez, C. J., and T. G. Mason, "Colloidal alphabet soup: Monodisperse dispersions of shape-designed lithoparticles," J. Phys. Chem. C 111(12), 4477-4480 (2007).

[62] Klingler, J. F., and H. M. McConnell, "Brownian motion and fluid mechanics of lipid monolayer domains," J. Phys. Chem. 97(22), 6096-6100 (1993).

[63] Choi, S. Q., Investigation of Rheological Properties of Fluid/Fluid Interfaces (University of California, Santa Barbara, 2011).

[64] Danov, K., R. Aust, F. Durst, and U. Lange, "Influence of the surface viscosity on the hydrodynamic resistance and surface diffusivity of a large Brownian particle," J. Colloid Interface Sci. 175(1), 36-45 (1995).

[65] Saffman, P. G., and M. Delbrück, "Brownian motion in biological membranes," Proc. Natl. Acad. Sci. U.S.A. 72(8), 3111-3113 (1975).

[66] Stone, H. A., and A. Ajdari, "Hydrodynamics of particles embedded in a flat surfactant layer overlying a subphase of finite depth," J. Fluid Mech. 369, 151-173 (1998).

[67] Happel, J., and H. Brenner, Low Reynolds Number Hydrodynamics (Martinus Nijhoff, The Hague, 1983).

[68] Fischer, T. M., "Comment on shear viscosity of Langmuir monolayers in the low-density limit," Phys. Rev. Lett. 92(13), 139603 (2004).

[69] Levich, V. G., and D. B. Spalding, Physicochemical Hydrodynamics (Prentice-Hall Englewood Cliffs, NJ, 1962).

[70] Lucassen, J., and R. S. Hansen, "Damping of waves on monolayercovered surfaces: I. Systems with negligible surface dilational viscosity," J. Colloid Interface Sci. 22(1), 32-44 (1966).

[71] Reichert, M. D., N. J. Alvarez, C. F. Brooks, A. M. Grillet, L. A. Mondy, S. L. Anna, and L. M. Walker, "The importance of experimental design on measurement of dynamic interfacial tension and interfacial rheology in diffusion-limited surfactant systems," Colloids Surf. A 467, 135-142 (2015).

[72] Crocker, J. C., and D. G. Grier, "Methods of digital video microscopy for colloidal studies," J. Colloid Interface Sci. 179(1), 298-310 (1996).

[73] Velankar, S. S., and D. Giles, "How do I know if my phase angles are correct?," Rheol. Bull 76, 8 (2007).

[74] Anguelouch, A., R. L. Leheny, and D. H. Reich, "Application of ferromagnetic nanowires to interfacial microrheology," Appl. Phys. Lett. 89(11), 111914 (2006).

[75] Samaniuk, J. R., and J. Vermant, "Micro and macrorheology at fluid fluid interfaces," Soft matter 10(36), 7023-7033 (2014).

[76] Kurtz, R. E., A. Lange, and G. G. Fuller, "Interfacial rheology and structure of straight-chain and branched fatty alcohol mixtures," Langmuir 22(12), 5321-5327 (2006).

[77] Kim, Y., J. Pyun, J. M. J. Frechet, C. J. Hawker, and C. W. Frank, "The dramatic effect of architecture on the self-assembly of block copolymers at interfaces," Langmuir 21(23), 10444-10458 (2005).

[78] Mason, T. G., J. Bibette, and D. A. Weitz, "Yielding and flow of monodisperse emulsions," J. Colloid Interface Sci. 179, 439-448 (1996).

[79] Djabbarah, N. F., and D. T. Wasan, "Dilational viscoelastic properties of fluid interfacesiii mixed surfactant systems," Chem. Eng. Sci. 37(2), 175-184 (1982).

[80] Brown, A. G., W. C. Thuman, and J. W. McBain, "The surface viscosity of detergent solutions as a factor in foam stability," J. Colloid Sci. 8(5), 491-507 (1953).
[81] Harvey, P. A., A. V. Nguyen, G. J. Jameson, and G. M. Evans, "Influence of sodium dodecyl sulphate and dowfroth frothers on froth stability," Miner. Eng. 18(3), 311-315 (2005).

[82] Petkov, J. T., K. D. Danov, N. D. Denkov, R. Aust, and F. Durst, "Precise method for measuring the shear surface viscosity of surfactant monolayers," Langmuir 12(11), 2650-2653 (1996).

[83] Barentin, C., C. Ybert, J.-M. Di Meglio, and J.-F. Joanny, "Surface shear viscosity of Gibbs and Langmuir monolayers," J. Fluid Mech. 397, 331-349 (1999).

[84] Ally, J., and A. Amirfazli, "Magnetophoretic measurement of the drag force on partially immersed microparticles at air-liquid interfaces," Colloids Surf. A 360(1), 120-128 (2010).

[85] Bouchama, F., and J.-M. Di Meglio, "Rheological studies of freely suspended soap films," Colloid Polym. Sci. 278(3), 195-201 (2000).

[86] Poskanzer, A. M., and F. C. Goodrich, "Surface viscosity of sodium dodecyl sulfate solutions with and without added dodecanol," J. Phys. Chem. 79(20), 2122-2126 (1975).

[87] Patist, A. R., T. Axelberd, and D. O. Shah, "Effect of long chain alcohols on micellar relaxation time and foaming properties of sodium dodecyl sulfate solutions," J. Colloid Interface Sci. 208(1), 259-265 (1998).

[88] Binks, B. P., "Particles as surfactants: similarities and differences," Curr. Opin. Colloid Interface Sci. 7(1), 21-41 (2002).

[89] Zell, Z. A., L. Isa, P. Ilg, L. G. Leal, and T. M. Squires, "Adsorption energies of poly (ethylene oxide)-based surfactants and nanoparticles on an air water surface," Langmuir 30(1), 110-119 (2013).

[90] Stancik, E. J., M. J. Widenbrant, A. T. Laschitsch, J. Vermant, and G. G. Fuller, "Structure and dynamics of particle monolayers at a liquidliquid interface subjected to extensional flow," Langmuir 18(11), 4372-4375 (2002).

[91] Park, B. J., J. P. Pantina, E. M. Furst, M. Oettel, S. Reynaert, and J. Vermant, "Direct measurements of the effects of salt and surfactant on interaction forces between colloidal particles at water-oil interfaces," Langmuir 24(5), 1686-1694 (2008).

[92] Wirth, C. L., E. M. Furst, and J. Vermant, "Weak electrolyte dependence in the repulsion of colloids at an oil water interface," Langmuir 30(10), 2670-2675 (2014).

[93] Park, B. J., J. Vermant, and E. M. Furst, "Heterogeneity of the electrostatic repulsion between colloids at the oil water interface," Soft Matter 6(21), 5327-5333 (2010).

[94] Bresme, F., and M. Oettel, "Nanoparticles at fluid interfaces," J. Phys.: Condens. Matter 19(41), 413101 (2007).

[95] Law, A. D., and D. M. A. Buzza, "Obtaining effective pair potentials in colloidal monolayers using a thermodynamically consistent inversion scheme," Langmuir 26(10), 7107-7116 (2010).

[96] Wurlitzer, S., H. Schmiedel, and T. M. Fischer, "Electrophoretic relaxation dynamics of domains in Langmuir monolayers," Langmuir 18(11), 4393-4400 (2002).

[97] Bouguet, J. Y., "Pyramidal implementation of the affine lucas kanade feature tracker description of the algorithm," Intel Corp. 5, 1-10 (2001).

[98] Jones, R. B., B. U. Felderhof, and J. M. Deutch, "Diffusion of polymers along a fluid-fluid interface," Macromolecules 8(5), 680-684 (1975).

[99] Rochefort, W. E., and S. Middleman, "Rheology of xanthan gum: Salt, temperature, and strain effects in oscillatory and steady shear experiments," J. Rheol. 31(4), 337-369 (1987). 